\title{
Old stellar Galactic disc in near-plane regions according to 2MASS: Scales, cut-off, flare and warp
}

\author{
M. López-Corredoira ${ }^{1,2}$, A. Cabrera-Lavers ${ }^{2}$, F. Garzón ${ }^{2,3}$, and P. L. Hammersley² \\ 1 Astronomisches Institut der Universität Basel, Venusstrasse 7, 4102 Binningen, Switzerland \\ 2 Instituto de Astrofísica de Canarias, 38200 La Laguna, Tenerife, Spain \\ 3 Departamento de Astrofísica, Universidad de La Laguna, Tenerife, Spain
}

Received 21 December 2001 / Accepted 7 August 2002

\begin{abstract}
We have pursued two different methods to analyze the old stellar population near the Galactic plane, using data from the 2MASS survey. The first method is based on the isolation of the red clump giant population in the color-magnitude diagrams and the inversion of its star counts to obtain directly the density distribution along the line of sight. The second method fits the parameters of a disc model to the star counts in 820 regions. Results from both independent methods are consistent with each other. The qualitative conclusions are that the disc is well fitted by an exponential distribution in both the galactocentric distance and height. There is not an abrupt cut-off in the stellar disc (at least within $R<15 \mathrm{kpc}$ ). There is a strong flare (i.e. an increase of scale-height towards the outer Galaxy) which begins well inside the solar circle, and hence there is a decrease of the scale-height towards the inner Galaxy. Another notable feature is the existence of a warp in the old stellar population whose amplitude is coincident with the amplitude of the gas warp.

It is shown for low latitude stars (mean height: $|z| \sim 300 \mathrm{pc}$ ) in the outer disc (galactocentric radius $R>6 \mathrm{kpc}$ ) that: the scaleheight in the solar circle is $h_{z}\left(R_{\odot}\right)=3.6 \times 10^{-2} R_{\odot}$, the scale-length of the surface density is $h_{R}=0.42 R_{\odot}$ and the scale-length of the space density in the plane (i.e. including the effect of the flare) is $H=0.25 R_{\odot}$. The variation of the scale-height due to the flare follows roughly a law $h_{z}(R) \approx h_{z}\left(R_{\odot}\right) \exp \left(\frac{R-R_{\odot}}{[12-0.6 R(\mathrm{kpc})] \mathrm{kpc}}\right)$ (for $R \lesssim 15 \mathrm{kpc} ; R_{\odot}=7.9 \mathrm{kpc}$ ). The warp moves the mean position of the disc to a height $z_{w}=1.2 \times 10^{-3} R(\mathrm{kpc})^{5.25} \sin \left(\phi+5^{\circ}\right) \mathrm{pc}$ (for $R \lesssim 13 \mathrm{kpc} ; R_{\odot}=7.9 \mathrm{kpc}$ ).
\end{abstract}

Key words. Galaxy: general - Galaxy: stellar content - Galaxy: structure - infrared: stars

\section{Introduction}

Star counts have been widely used for the study of Galactic structure (see Paul 1993), and are becoming increasingly important with the appearance of wide area surveys in the last decades (see for example Bahcall 1986; Majewski 1993; Reid 1993; Garzón et al. 1993; Price 1988). The use of progressively larger and more sensitive samples combined with detailed models of stellar galactic distribution (for example Bahcall \& Soneira 1980; Wainscoat et al. 1992) has overcome most of the original uncertainties and difficulties in the analysis and interpretation of star count data. The advent of NIR detector arrays has permitted the detailed exploration of the stellar structure in hitherto hidden areas of the Milky Way, such as the Galactic Plane and Bulge (Eaton et al. 1984; Habing 1988; Garzón et al. 1993; Hammersley et al. 1994; Ruphy et al. 1996; LópezCorredoira et al. 2000; Epchtein 1997; Skrutskie et al. 1997). This is because at these wavelengths interstellar extinction is significantly less when compared to the visible, while keeping the individual stellar contribution to the observed flux.

Send offprint requests to: M. López-Corredoira, e-mail: martinlc@astro.unibas.ch
There are, however, still controversial or totally unknown parameters in the description of the detailed stellar structure. Some of these are concerned with the radial and vertical distribution of the Galactic disc, and its specific morphology. Several radial scale-lengths have been determined by different groups, even when using the same data sets. As a general trend, the shorter scale-lengths are found for the surveys using longer wavelengths (Kent et al. 1991), although it could equally be that different source type or regions of the Galaxy are being examined. There is far less debate on the vertical scale-height, with the canonical value for the old population in the Solar vicinity being about 300 pc. Still more work is needed, however, to differentiate between the distribution being an exponential or the $\operatorname{sech}^{2}$ (van der Kruit 1988). Finally, features in the stellar disc like internal (Freudenreich 1998; López-Corredoira et al. 2001) or external (Habing 1988; Ruphy et al. 1996) cut-offs radii, flares (Kent et al. 1991; Alard 2000), warps (Djorgovski \& Sosin 1989; Freudenreich 1998; Porcel \& Battaner 1995; Alard 2000; Drimmel \& Spergel 2001) and/or local corrugations of the galactic plane (Hammersley et al. 1995) are less well studied, and are more controversial. 
In this paper we will address some of the above topics by analyzing the shape of the stellar Galactic disc in two ways. We will first extract a single stellar type by using the deep NIR color-magnitudes diagrams (CMD) from the 2MASS survey (Skrutskie et al. 1997). The analysis will be based on the red clump giants, whose relatively high density and bright absolute magnitude give rise to an identifiable feature in the CMDs. Hence, the distance and extinction to each star can be directly determined only assuming the absolute magnitude and color of the sources. As a second approach, we will use the star counts, also taken from 2MASS, and the results will then be compared with those obtained from the red clump method. Both methods will be used to examine the scale-length, scale-height, flare, outer disc cut-off and warp.

The data for this work has been taken from the 2nd release of the 2MASS project (Skrutskie et al. 1997, http://www.ipac. caltech. edu/2mass/releases/docs.html). We have made use of the available data in that second release for near plane regions in the outer Galaxy $\left(45^{\circ}<l<315^{\circ}\right)$.

\section{Model of a non-warped Galactic disc}

In order to help to interpret the 2MASS star counts a simple model of the density distribution in the disc was built.

The Galactic disc is assumed to be an exponential distribution whose star density is:

$\rho(R, z)=\rho_{\odot} \exp \left(-\frac{R-R_{\odot}}{h_{R}}\right) \exp \left(-\frac{|z|}{h_{z}(R)}\right) \frac{h_{z}\left(R_{\odot}\right)}{h_{z}(R)}$,

$h_{z}(R)=h_{z}\left(R_{\odot}\right) \exp \left(\frac{R-R_{\odot}}{h_{R, \text { flare }}}\right)$,

where $\rho_{\odot}$ is the space density on the plane in the neighborhood of the Sun.

Hence the density falls exponentially with the galactocentric radius $R$ (scale-length $h_{R}$ ), and with the height $z$. We have preferred an exponential scale-height rather than a $s e c h^{2}$, as Hammersley et al. (1999) shows that a sech ${ }^{2}$ law did not give a good fit to the TMGS star counts near the plane. The effect of the flare is to increase the scale-height of the sources in the outer Galaxy, hence the scale-height $\left(h_{z}(R)\right)$ is dependent on $R$. We have assumed that the total number of stars at a specific galactocentric radius remains the same as it would have been without the flare and so all the flare is doing is to distribute the sources further from the plane. In the model this is included by having the scale-height increase exponentially with galactocentric radius and then have a normalizing factor $\left(\frac{h_{z}\left(R_{\odot}\right)}{h_{z}(R)}\right)$ for the density. Whilst this is not the first model to include a flare, it is the first to use an exponential rather than a linear flare.

Another way of expressing the density is:

$\rho(R, z)=\rho_{\odot} \exp \left(-\frac{R-R_{\odot}}{H}\right) \exp \left(-\frac{|z|}{h_{z}(R)}\right)$,

where

$H=\left(\frac{1}{h_{R}}+\frac{1}{h_{R, \text { flare }}}\right)^{-1}$, i.e. $H$ is the equivalent scale-length which is the combination of the intrinsic scale-length of the disc with that of the flare. Written in this form the equivalent scale-length takes the place of the normalizing factor. The flare increases with $R$ and so removes proportionally more stars from the plane with increasing distance. The net effect is that on the plane the star density falls far more rapidly with increasing galactocentric distance than the intrinsic scale-length predicts. Hence, $h_{R}$ is the scale-length of surface density, and $H$ is the scale-length of the space density in the plane.

We have assumed a distance from the Sun to the Galactic center to be $R_{\odot}=7.9 \mathrm{kpc}$ (López-Corredoira et al. 2000).

Therefore, this model contains only three free parameters $\left(h_{R}, h_{z}\left(R_{\odot}\right), h_{R \text {,flare }}\right)$ whose determination is carried out by minimizing the $\chi^{2}$ of the fit in the following way.

For a given set of parameters, the space density on the plane in the neighborhood of the Sun can be determined using the formula

$\rho_{\odot}=\frac{\sum_{i} \frac{d_{i} t_{i}}{\sigma_{i}^{2}}}{\sum_{i} \frac{t_{i}^{2}}{\sigma_{i}^{2}}}$,

where $d_{i}$ is the measured density in each region, $\sigma_{i}$ the poissonian errors in the counts and $t_{i}$ is the model prediction with $\rho_{\odot}=1$. Hence, for each set of model parameters we calculate $\rho_{\odot}$ through Eq. (5), and after that we calculate $\chi^{2}$ for that particular fit. Once we have calculated all the $\chi^{2}$ for all sets in the parameter space, we select the one which gives a minimum $\chi^{2}$. The errors are due to the errors in the counts (assumed to be poissonian) and errors coming from the calculation.

The density equation can be further simplified on plane ( $b \approx 0$ ), as long as $|z| / h_{z}(R)<<1$ along the whole line of sight:

$\rho(R, z=0)=\rho_{\odot} \exp \left(-\frac{R-R_{\odot}}{H}\right)$.

If the external disc were truncated, then another parameter that could be added is the radius of the cut-off $R_{\text {cut-off }}$. It will be shown in the analysis presented here (Sect. 3.5), however, that there is no evidence for a cut-off to at least $R=15 \mathrm{kpc}$, and so this parameter will be unnecessary in the fits. Beyond $R \approx$ $15 \mathrm{kpc}$, there are very few stars detected and so very little can be said.

We have ignored the contribution of the spiral arms since in the outer Galaxy their contribution to the star count is small and even then it will be at the brightest magnitudes being considered here, giving negligible contribution for $m_{K}<14.0$.

Other possible additions to the standard disc model are large scale warps and the presence of the thick disc. The warp will be considered separately in Sect. 5 .

The thick disc (Gilmore \& Reid 1983; Buser et al. 1998, 1999; Ojha 2001) will not be studied here as a separate population since the analysis presented here is not sensitive to thick disc, as regions in general near the plane are used where its relative contribution is small. It will be shown in Sects. 3.5 and 4 that the thick disc could constitute as much as a $10 \%$ of the total low latitude sources selected in this paper, so the derived scale-height will be somewhat larger than would be the case 
for the thin disc alone. Here we will use the term "old disc" as the average structure traced by the old disc stars, including any thick disc component (mean height around $300 \mathrm{pc}$ ). The determined mean scale-height might be larger at higher latitudes if there is a thick disc.

\section{Using CMDs to obtain the parameters of the disc}

\subsection{The selection of giants}

We have built $\left(J-K, m_{K}\right)$ CMDs from the 2MASS data set for selected regions of the sky (Fig. 1). We have taken the data "as is", without further processing. The data have been grouped in regions covering between 2 and 5 squared degrees on the sky (see Sect. 3.4 for details).

The position on a CMD of a star is determined from its absolute magnitude, intrinsic color, distance and extinction. Any horizontal displacement is caused by extinction alone, and hence if the intrinsic color of the source is known, the total extinction can be directly determined from the measured color. The vertical motion, however, is caused by both distance (doubling the distance makes the source 1.5 mag fainter) and extinction. So if the absolute magnitude of the source is already known, the extinction can be determined from the reddening and hence the distance is directly determined (see formulae in Sect. 3.2). When there are a number stars of the same spectral class (i.e approximately the same absolute magnitude and color) but at different distances from the Sun, these will be situated along a line on the CMD. Increasing the distance shifts the star to a fainter magnitude, while extinction by itself shifts the stars diagonally. Hence, combining these two effects would mean that sources from a single spectral class will form a line (or stripe) on a CMD. In areas of the Galaxy where extinction is low this line will be almost vertical whereas for lines of sight into the inner Galaxy, the line will have a considerable angle as the extinction becomes the dominant effect.

On a color-absolute magnitude diagram (CAMD), the majority of the stars lie in the main sequence or giant branch. When there is a wide range of distances being sampled, each part of the CAMD produces its own stripe on a CMD and so the overall effect is more of a broad smear rather than a well defined line. By far the most common type of giant, however, are the early $K$ giants or red clump giants. These produce a very well defined grouping, with a density far higher than the surrounding areas, on a visible CAMD (e.g. Hoeg et al. 1997). On an IR CAMD (e.g. Cohen et al. 2000) the grouping is even tighter. For $V-$ [8.3] CAMD the total spread of the red clump is about 0.5 in color and $1 \mathrm{mag}$ in absolute magnitude. There are sufficient of these sources to produce a clearly recognizable feature in a CMD, and this was used in Hammersley et al. (2000) to examine lines of sight into the inner Galaxy. The luminosity function derived in Hammersley et al. (2000) shows that at the peak of the red clump there are about 10 times the numbers of sources as there are either 0.8 mag brighter or fainter, and the sigma in absolute magnitude is about $0.3 \mathrm{mag}$. The $M_{K}$ of the center of the red clump is -1.65 , although there is a possible error of about $0.1 \mathrm{mag}$. Using the tables in
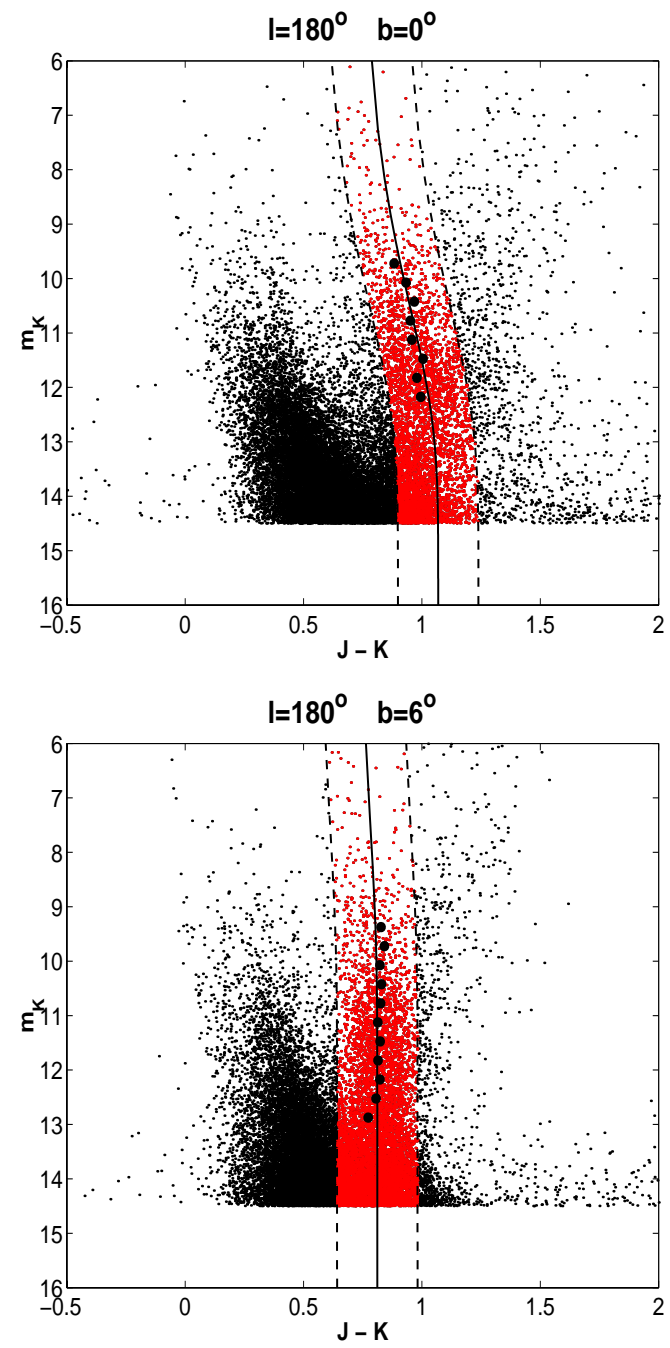

Fig. 1. Color-magnitude diagrams for two studied fields. The higher extinction causes a slight broadening in the $K$-giants strip for in-plane regions. Maxima obtained by a Gaussian fitting to star distribution in the red clump strip. The solid line shows the fitted trace that we assign to red clump giants population. The dashed lines show the limits for the red clump giants extraction within a width of 0.4 mag.

Wainscoat et al. (1992) an $M_{K}=-1.65$ corresponds to a K2III and hence the $J-K$ color is $(J-K)_{0}=0.75$.

Figure 1 shows the CMD for two of the fields used in this study. The red clump stripe can be clearly seen. In order to isolate the red clump sources, we have made histograms of horizontal cuts (i.e. in color) through the CMDs, varying the $m_{K}$ range used. A Gaussian was then fitted to histogram (i.e. the region containing the red clump giants) to determine the position of the peak in each horizontal cut. Stars lying well to the left of the maxima are almost totally dwarfs, as there are very few giants with colors bluer than the red clump stars. Stars lying to the right will be predominantly the $M$ giants and AGBs, at least brighter than $m_{K}=13$.

In order to simplify the parameter fitting it will be assumed that the red clump giants all have $M_{K}=-1.65$ (i.e. luminosity function becomes a Dirac delta function) instead of a more realistic case with some dispersion (see in Sect. 3.3 a discussion about this approximation). The peak of the histogram is 
used to trace the red clump giants empirically. However, it can be seen in Fig. 1 that at fainter magnitudes the effect of the $K$ dwarf population becomes significant and swamps the red clump sources. Hence, this limits the method to $m_{K}<13.0$. Figure 1 show the position of the peaks on each of the CMDs. Using the fitted trace for the red clump giants extinction along the line of sight for each field can be determined (see Sect. 3.2).

The red clump giants can be directly extracted using the fitted trace. Sources with a $(J-K)$ within 0.2 mag of the center of the fitted red clump line were extracted, and the star counts were then obtained. The width was determined after a number of trials and is a compromise between the necessity to avoid other stars types but include most of the red clump giants. If too small a width were chosen then any error in the determination of the position peak would lead to a loss of sources at that point and so reduce the measured density. Using this width, most of the red clump giants for low extinction regions will be extracted. However, where the extinction is somewhat patchy (for instance in negative latitudes) the strip broadens and a significant number of stars are lost. Therefore, those regions have been excluded from the analysis.

One of the main strengths of this method is that it is empirical and the only assumption being made is that the absolute magnitudes of all of the sources being extracted is fixed. If, however, we had taken a theoretical trace for the red clump giants using a model and isolated the stars around this, then any difference between the predicted and actual extinction would directly lead to errors in the extracted counts. Another advantage of this method is that the spatial information is obtained directly from the counts. The position of the sources can be determined directly from the magnitude and extinction without the need to resort to models.

The error analysis presented in the following sections will only include the random errors in the fitting and not the systematic errors outlined in this section. The error in the determination of the absolute magnitude is taken to be $0.1 \mathrm{mag}$, which corresponds to a distance error of $5 \%$. The possible error in $(J-K)_{0}$ only affects the calculation of the extinction, as the peaks of the color histograms are derived directly from the data. An error of $0.1 \mathrm{mag}$ in $(J-K)_{0}$ would lead to an error of $0.07 \mathrm{mag}$ in the extinction (see Eq. (8)) and hence an error of $3 \%$ in distance. The errors in assuming that the luminosity is a delta function are also of the order $5 \%$. To these should be added the errors in the 2MASS zero points or the assumed extinction coefficients although these are likely to be small. The photometric error in 2MASS sources with $m_{K}<13$ is $\sim 0.03$ mag., however the error is much lower when using the mean magnitude in a bin with tens of stars. Similarly, the error in the 2MASS-color is negligible for of the same reason. Hence, as well as the stated error there will be a systematic error in the distances, but this should be under $10 \%$.

\subsection{Stars density. Disk scale-length}

Using the red clump star counts, we can directly obtain the star

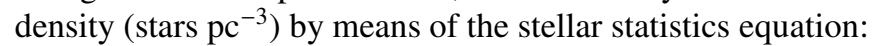

$A(m)=w \int_{o}^{\infty} r^{2} D(r) \phi(M) \mathrm{d} r$ where $A(m)$ is the number of stars per unit area of solid angle $w$ at $m$ in interval $\mathrm{d} m$, and $\phi(M)$ is the luminosity function. The bins used in this paper have $\mathrm{d} m=0.1$ in the range $m_{k}=8.5$ to $m_{k}=13.0$.

As has been already discussed, we have assumed $M_{K}=$ -1.65 and an intrinsic color $(J-K)_{0}=0.75$ for a red clump giants. The extinction $A_{K}\left(m_{k}\right)$, to a distance $r$, can be determined by tracing how $(J-K)$ of the peak of the red clump counts changes with $m_{K}$. The extinction is calculated for any $m_{K}$ using the measured $(J-K)$ of the peak, the intrinsic mean color of the stars in the apparent magnitude bin $(J-K)_{0}$, and the color excess definition and the interstellar extinction values for $A_{J}$ and $A_{K}$, as given by Rieke \& Lebofsky (1985):

$A_{K}=\frac{(J-K)-(J-K)_{0}}{1.52}$.

Since we have assumed that red clump giants have an absolute magnitude of $M_{K}=-1.65, \phi(M)$ is replaced by a delta function. Furthermore, by choosing apparent-magnitude intervals of $0.1 \mathrm{mag}(\delta m)$ and the related distance intervals $(\delta r)$, the star density can be directly determined using:

$r=10^{\frac{m_{K}-M_{K}+5-A_{K}(r)}{5}}$,

$D(r)=\frac{A(m) \delta m}{w r^{2} \delta r}$

The galactocentric mean distance of the stars per bin $(R)$ is calculated using the measured mean distance $(r)$ in the following equation:

$R=\sqrt{R_{0}^{2}+(r \cos b)^{2}-2 r \cos b R_{0} \cos l}$.

With this method, we are inverting the star counts of the red clump population to obtain the stellar density along the line of sight. This is a simple and direct method and although some assumptions and approximates are required, these can be justified. Another method of inversion of star counts is presented in López-Corredoira et al. (2000) which solved for the both density and luminosity function. However, this method only works for the bulge and would not be appropriated for the disc.

\subsection{Testing the method}

In order to to verify that the proposed method is correct a number of tests were made.

\subsubsection{The difference between the real red clump distribution and a Dirac's delta}

To further explore the form of red clump, we have calculated the distribution of $K$ magnitudes and $(J-K)_{0}$ colors using the "SKY" model, but updating the density function of the giant populations (Wainscoat et al. 1992; Cohen M., private communication). In Fig. 2, the part of the luminosity function pertaining to the G5 to M0 giants is shown. The right hand plot shows the predicted form of the $(J-K)$ histogram at $m_{K}=+11$ (but with no extinction) along a line of sight towards the anticenter. In both plots the predominance of the red clump giants is evident. 

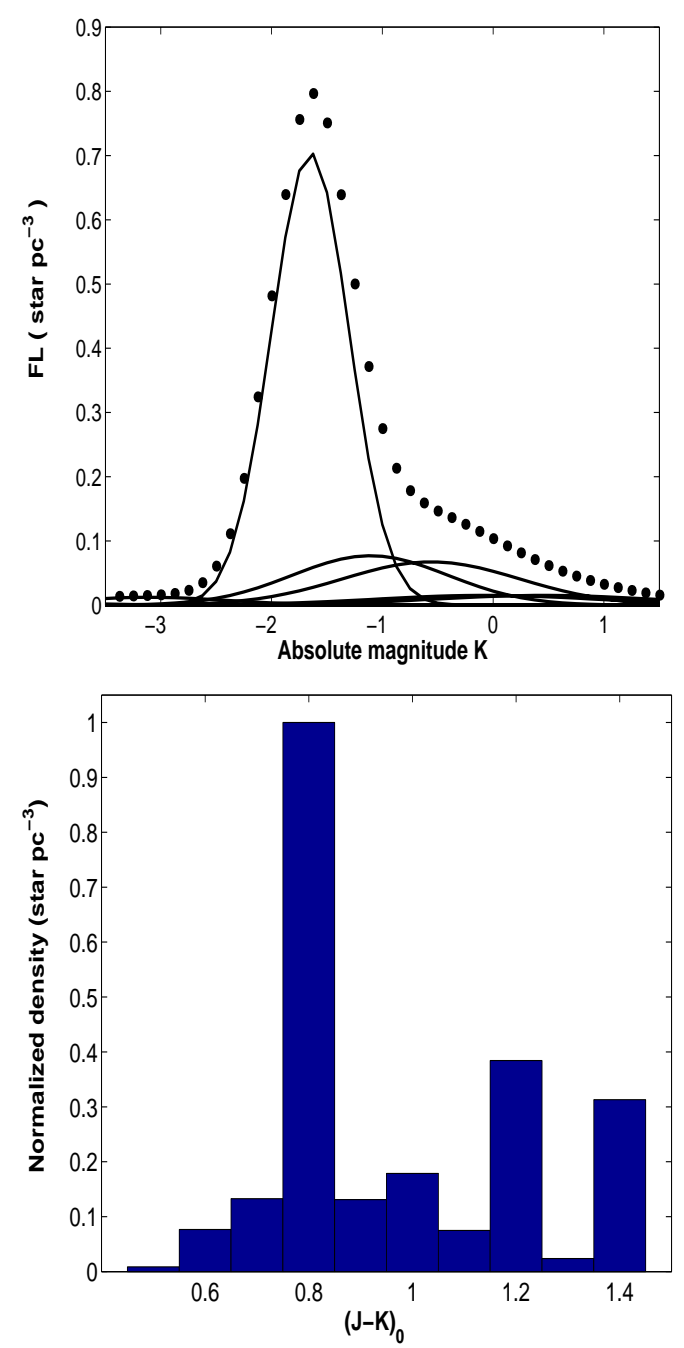

Fig. 2. Distribution of $K$ absolute magnitudes and $(J-K)_{0}$ colors at $m_{K}=11$ along a line of sight towards the anti-center corresponding to the sum of all giant types in the disc according to the updated "SKY" model (Wainscoat et al. 1992; M. Cohen, private communication). The maxima of these plots corresponds the red clump, which are predominant in the giant population.

In order to determine the effect of using a Dirac delta as the luminosity function for the red clump, we have carried out a simple calculation. The red clump giants are assumed to have a Gaussian dispersion in magnitude with $\sigma=0.3$. From Eqs. (7) and (10), the density in the direction of the anticenter can then be written as:

$$
\begin{aligned}
\rho^{\prime}\left(R-R_{\odot}\right)= & \frac{5}{\ln (10) \sqrt{2 \pi} \sigma} \frac{1}{\left(R-R_{\odot}\right)^{3}} \\
& \times \int_{0}^{\infty} \mathrm{d} r r^{2} \rho(r) \mathrm{e}^{-\frac{1}{2}\left(\frac{5}{\sigma} \log \frac{\left(R-R_{\odot}\right)}{r}\right)^{2}},
\end{aligned}
$$

where $\rho^{\prime}$ is the density derived with the method developed in this section (see Sect. 3.2), while $\rho$ is the real density distribution. Using Eq. (6) with $H=2.0 \mathrm{kpc}, \rho_{\odot}=1$, gives the result plotted in Fig. 3. The difference between the two curves is the error introduced by the approximation, hence it is negligible. The scale-length that is determined assuming $M_{K}=-1.65$ for all of the sources is $H^{\prime}=1.96 \mathrm{pc}$, i.e. the error in the calculation

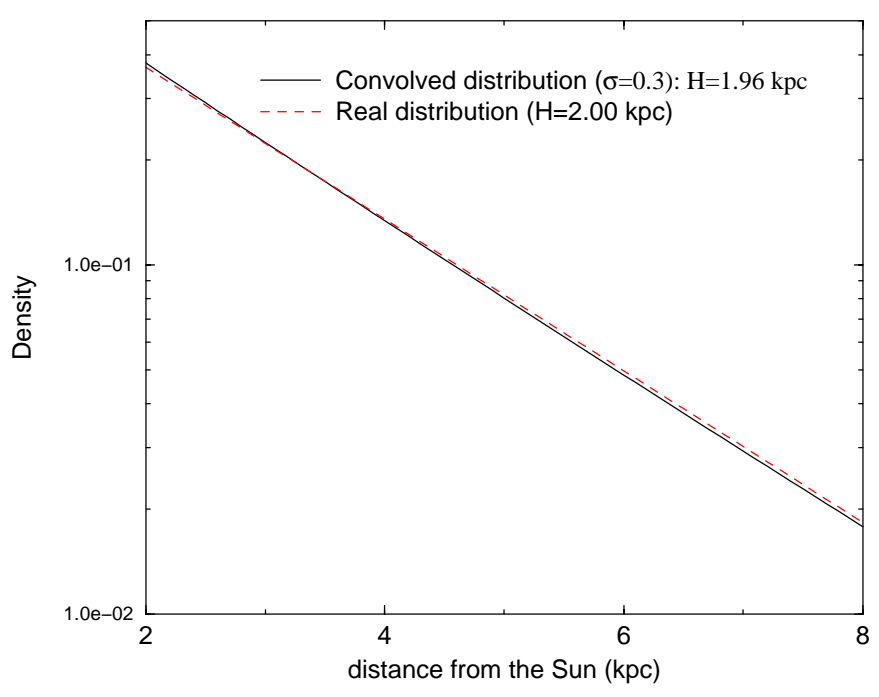

Fig. 3. Comparison between a real density $\rho=\mathrm{e}^{-\frac{d}{H}}$ and the convolved distribution of Eq. (12) obtained under the assumption of Dirac delta luminosity function instead of the real Gaussian distribution with $\sigma=0.3$.

of the scale-length is around $2 \%$. Therefore, the simplification is justified and it does not produce significative differences.

\subsubsection{Can the cut-off be masked by contamination of low luminosity giants with the same color than the red clump?}

One critical aspect of the method is the assumption that there are only red clump sources being extracted (i.e. $M_{K}=-1.65$ ). However, the CAMD presented in Cohen et al. (2000, their Fig. 4), shows that there are lower luminosity sources with the same color as the red clump. (The range of $(J-K)$ used for extracting the red clump corresponds to a $V-[8.3]$ of about 1.2). The magnitude limit for the CAMD is about $m_{[8.3]}=6.5$ and so in the 100 pc plot of Fig. 4 in Cohen et al. (2000), the source count is complete for all absolute magnitudes brighter than 1.5. There are some higher luminosity sources with the same color and the red clump, but these are relatively few and can be ignored. However, there are significant numbers of lower luminosity giants. These giants will have $+2>M_{K}>-1.4$ and could mask a cut off.

In order to gauge the effect of these sources on the method, a simple luminosity function was built which had half of the giants with $-1.45>M_{K}>-1.85$ and the other half of giants with $+2>M_{K}>-1.4$. This is probably an overestimate of the importance of the lower luminosity sources and so should be considered a worse case scenario. We have carried out the same type of convolution in the anticenter of the previous subsubsection but with this luminosity function, and we have used a truncated exponential disc. The result is shown in 4 .

As can be observed, the only significant change is in the amplitude because half of the stars with $M_{K}=-1.65$ have been lost. However, this is not important as the total number of the giants is not used in the analysis. Also the derived scale-length changes by $7 \%$. The cut-off, however, is not masked as there is a clear change of slope in $\log \rho$ around $d \approx 5 \mathrm{kpc}$. Hence, 


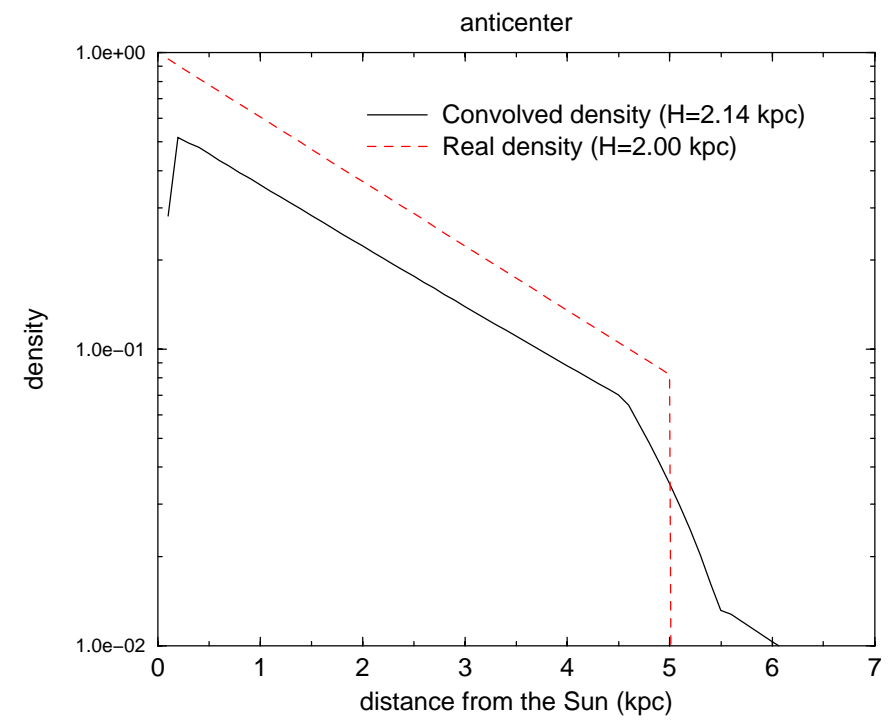

Fig. 4. Comparison between a real density $\rho=\mathrm{e}^{-\frac{d}{H}}$, truncated at $R=12.9 \mathrm{kpc}$ (distance from the Sun: $5 \mathrm{kpc}$ ) and and the convolved distribution obtained under the assumption of Dirac delta luminosity function instead of the real distribution with $50 \%$ of the stars with $M_{K}=-1.65$ and $50 \%$ of the stars between $+2>M_{K}>-1.4$ (lower luminosity giants).

if there were a cut-off at about $13 \mathrm{kpc}$ it should still be visible even with the presence of possible lower luminosity giants. As will it be shown below (Figs. 7 and 9), we do not observe any change of slope in $\log \rho$, so the absence of cut-off seems clear and cannot be criticized as an effect of the contamination of low luminosity giants.

\subsubsection{Dwarf contamination}

Another critical aspect that must be considered is the dwarf contamination. There is an important contribution to the stars counts from dwarf stars for faint apparent magnitudes $\left(m_{K}>\right.$ 14; Fig. 1). The selection method used here cannot separate between these two star populations at these magnitudes, as the main sequence and the $K$-giant strip overlap. In order to estimate the dwarf contamination for $m_{K}<13.0$, the luminosity and densities functions of the giants and dwarf stars from the model of Wainscoat et al. (1992) were used to calculate the predicted ratio between dwarf and the total of giants plus dwarfs in the counts. The result is shown in Fig. 5. It is clear that for faint apparent magnitudes $\left(m_{K} \simeq 14\right)$ the dwarf contribution is important, accounting for almost $50 \%$ of the total counts. However, the contribution decreases at brighter magnitudes such that for $m_{K}<13$ less than $\sim 10 \%$ of the stars are dwarfs. This was calculated for off-plane regions, where the extinction is almost negligible. It is expected that for regions close to the Galactic plane, where the extinction is higher, the dwarf contamination will be even less, because the reddening will shift the giants to the right whilst the dwarfs will be less affected, and so the dwarf-giant separation is improved. Hence, here we will only use the red clump star counts for $m_{K}<13.0$, where the dwarf contamination is small.

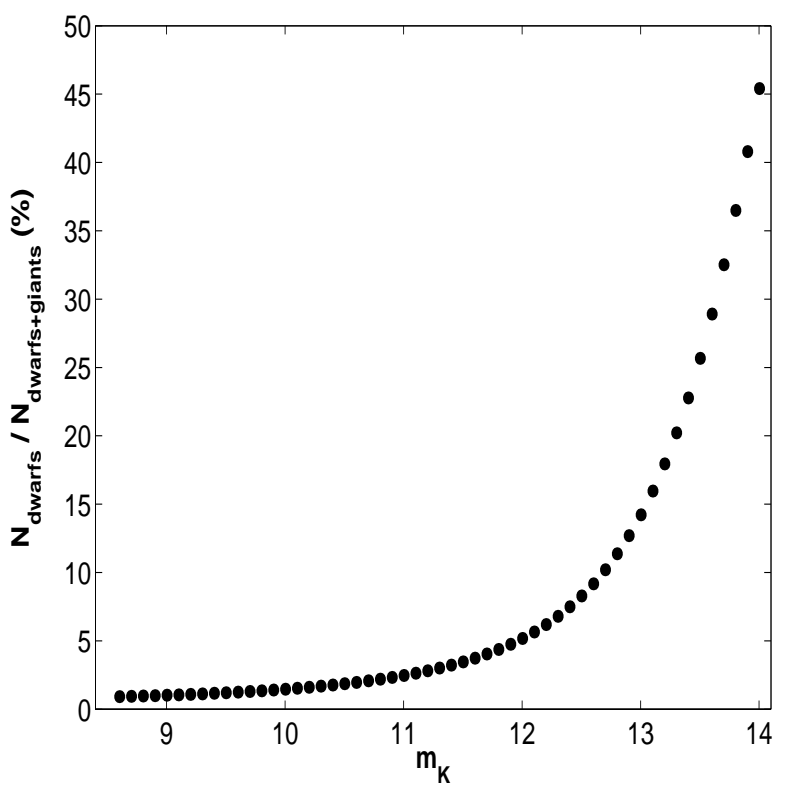

Fig. 5. Percentage of predicted dwarf stars in the total star counts extracted from the color-magnitude diagram for $l=180^{\circ}, b=0^{\circ}$.

\subsubsection{The ambiguity between reddening and metallicity effects for the clump giants}

When the metallicity decreases the stars become bluer, and hence the reddening could be underestimated.

We have assumed that the stellar populations throughout the disc is homogeneous and therefore, there are no large gradients in the metallicity. However, even in the case of small metallicity variations (Cameron 1985, has measured a radial metallicity gradient of $-0.11 \mathrm{dex} \mathrm{kpc}^{-1}$ ), the change to the result is small as the red clump color is practically independent of age and metallicity (Sarajedini et al. 1995). The variations are limited within $\Delta(B-V)_{0}<0.05$ (Sarajedini et al. 1995), which means $\Delta(J-K)_{0}<0.022$, i.e. an error in the extinction less than 0.015 , which is negligible.

\subsection{Selected regions}

Twelve fields were chosen from the available 2MASS data, which are summarized in Table 1 . In each field a large area of sky was covered (between 2 and 5 square degrees) as a large number of stars are needed to determined the color of the peak of the red clump distribution. The high number of red clump giants also minimizes the poissonian noise in the star counts and so improves the subsequent fitting of the density distributions. The fields were $5^{\circ}$ in longitude by $1^{\circ}$ in latitude, although the actual area covered depends on the available 2MASS data within each region. The star density is changing far more rapidly in latitude than longitude so it is important not to include a wide spread of latitudes in each region. Positive latitude regions were selected because these are less affected by patchy extinction, which causes a significant dispersion of the red clump strips. We have only used regions $150^{\circ}<|l|<225^{\circ}$ so as to avoid the northern and southern warps, which produce large asymmetries between positive and negative latitudes (see 
Table 1. Selected regions used with the red clump extraction.

\begin{tabular}{ccccc}
\hline \hline$l(\mathbf{d e g})$ & $b(\mathbf{d e g})$ & ${\boldsymbol{a r e a ~}\left(\mathbf{d e g}^{2}\right)}$ & $N_{\text {stars }}\left(m_{k}<14.5\right)$ & $N_{\text {red clump, } F W H M=0.4 \mathrm{mag}}\left(m_{K}<13\right)$ \\
\hline 180 & 0 & 3.84 & 68492 & 2588 \\
180 & 3 & 5 & 98473 & 4881 \\
180 & 6 & 5 & 64366 & 3777 \\
180 & 9 & 5 & 49614 & 7161 \\
180 & 12 & 5 & 39017 & 5191 \\
220 & 0 & 5 & 111788 & 5100 \\
220 & 3 & 3.88 & 69659 & 3756 \\
220 & 6 & 5 & 75411 & 4024 \\
220 & 9 & 4.99 & 54672 & 6555 \\
220 & 12 & 3.55 & 30867 & 3992 \\
155 & 0 & 2.07 & 24189 & 1225 \\
165 & 0 & 4.22 & 71731 & 3765 \\
\hline
\end{tabular}

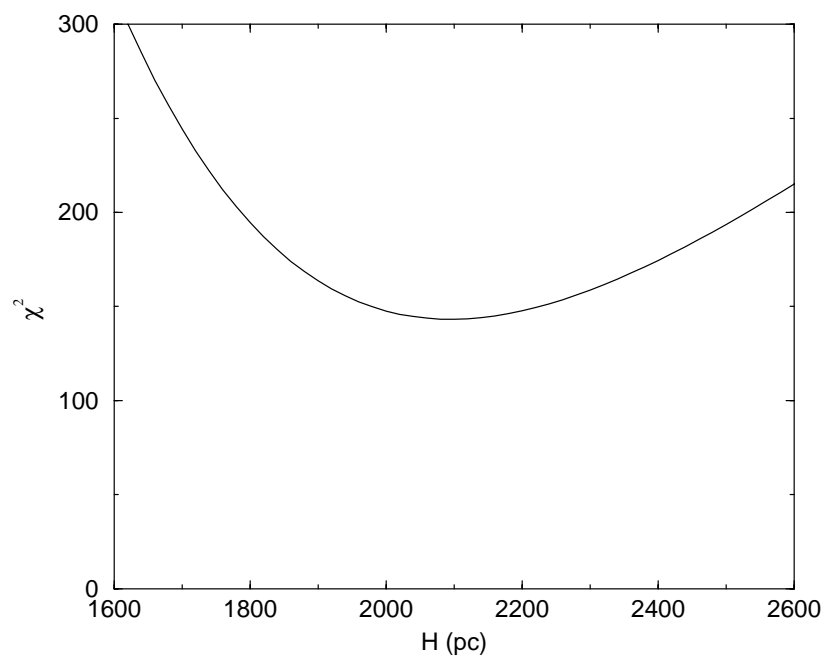

Fig. 6. $\chi^{2}$ vs. $H$ of the fit of red clump star density in the plane of the model (6).

Sect. 5). Furthermore, the lines of sight in $45^{\circ}<|l|<90^{\circ}$ travel for large distances at approximately the same galactocentric distance before reaching the outer disc, which would increase the error in obtaining the scale-length.

The main characteristics of the chosen fields are summarized in Table 1. Columns (1) and (2) give the Galactic coordinates of the center of the field. Column (3) is the total area in the field which has 2MASS data. The total number of stars with $m_{k}<14.5$ is given in Col. (4), and finally Col. (5) gives the total number of red clump giant stars extracted from the color-magnitude diagram, with $m_{k}<13$.

\subsection{Results}

A sequential fitting procedure has been used to obtain the model parameters for Eqs. (1) and (2) (or Eqs. (3) and (4)). First, to restrict the number of free parameters we have used only the data at $b=0$ to obtain the radial scale-length $H$ (i.e., Eq. (6)), so that there would be no dependence on height. This scale-length is then used in the off-plane regions to derive the values of the scale-height $\left(h_{z}(R)\right)$ and flare $\left(h_{R \text {,flare }}\right)$.

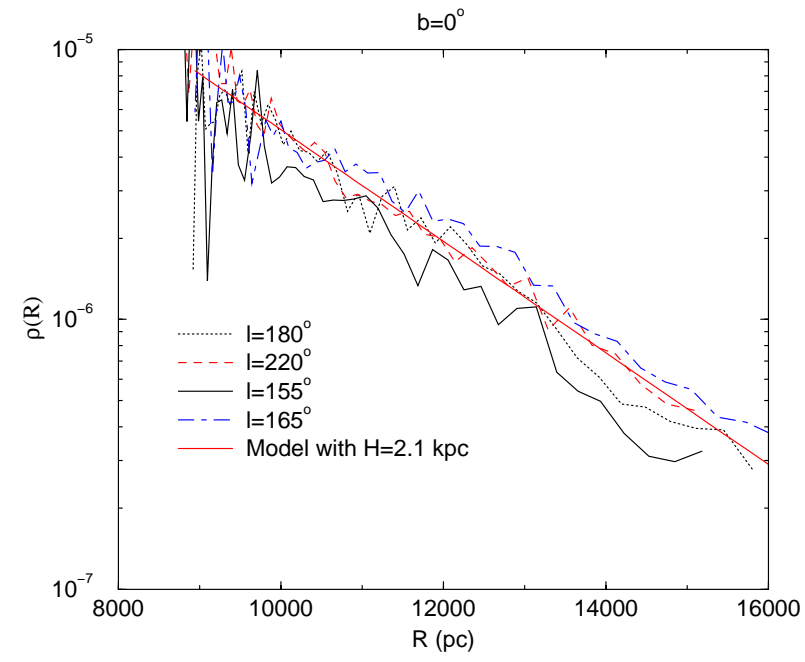

Fig. 7. Fit of red clump star density in the plane of the model (6).

An exponential disc (Eq. (6)) was fitted to the data with $m_{K} \leq 13.0$ in the plane for longitudes of $155^{\circ}, 165^{\circ}, 180^{\circ}$ and $220^{\circ}$. The value of $H$ which gives the minimum $\chi^{2}\left(\chi^{2}=143.2\right.$ for a number of data points $N=184$, see Fig. 6) and assuming that the errors for each data point are poissonian was for:

$H=2.10_{-0.17}^{+0.22} \mathrm{kpc}$.

As described in Sect. 3.1, the red clump sources are extracted from the general star counts by means of the $(J-K)$ CMD. Inserting these number counts in Eq. (5), with the help of Eq. (6) with the calculated value for $H$, we derive a density for these sources in the solar neighborhood $\rho_{\odot \text {,red clump }}=$ $1.31 \times 10^{-5} \mathrm{stars}_{\mathrm{pc}}{ }^{-3}$. The disc model with the optimum value of $H$ is shown in Fig. 7 together with the density distribution of the red clump stars derived as explained in Sect. 3.2. The small differences in the density for the different lines of sight stem presumably from the differences in the patchiness of the extinction, which spreads out the red clump giants, or contamination. Even so, it is noticeable how well the analytical function matches the data.

Using the value of $H$ determined above with off-plane regions at $l=180^{\circ}$ and $l=220^{\circ}$, allows the disc scale-height and the flare scale-length to be examined. As before, the disc 


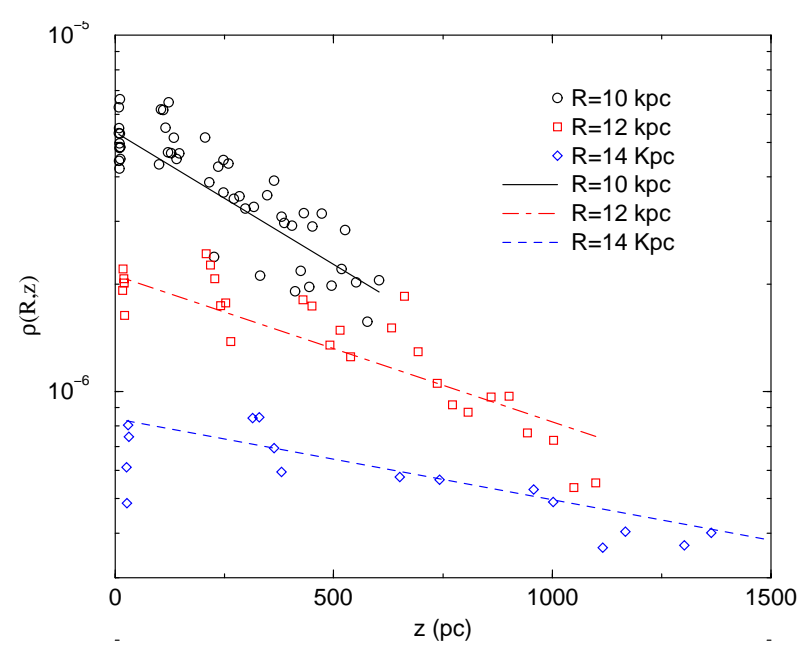

Fig. 8. The red clump star density obtained from the data and the lines showing a model (3) with $H=2.1 \mathrm{kpc}, h_{z}\left(R_{\odot}\right)=310 \mathrm{pc}, h_{R \text {,flare }}=$ $3.4 \mathrm{kpc}$. Note that the data show a slower decrease of density for larger $R$, and this fact can be modeled with the flare $h_{z}=580 \mathrm{pc}, 1055 \mathrm{pc}$, $1905 \mathrm{pc}$ respectively for $R=10,12,14 \mathrm{kpc}$ (the data belong to a range of $0.5 \mathrm{kpc}$ centered in these galactocentric radii).

model of Eqs. (3) and (4) are matched to the number counts by minimising the $\chi^{2}$ of the fit, being now the free parameters

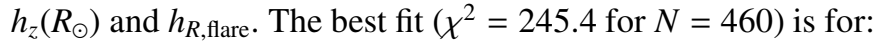

$h_{z}\left(R_{\odot}\right)=310_{-45}^{+60} \mathrm{pc}$,

$h_{R \text {,flare }}=3.4 \pm 0.4 \mathrm{kpc}$.

The errors are calculated for $H=2.10 \mathrm{kpc}$ and other values of $H$ could slightly change the error ranges. The stated errors are determined to be changing the parameters such that $\chi^{2}$ becomes a factor $f$ larger than the minimum (which corresponds to a $68 \%$ confidence level and is specified in statistics manuals). The correlations between the different parameters do not significatively change the errors. Figures 8 and 9 show the comparison of this model with the observational data at different heights above the plane. Since the heliocentric distance to the extracted stars can be measured (see Sect. 3.2), their distance from the Galactic plane is also known. Both figures show that there is an increase in scale-height with increasing $R$. Furthermore, in Fig. 8, the data show a slower decrease of stellar density with $z$ at larger $R\left(h_{z}=580 \mathrm{pc}, 1055 \mathrm{pc}, 1905 \mathrm{pc}\right.$ for $R=10,12,14 \mathrm{kpc}$ respectively). Both of these effects can only be produced by a flare.

This result cannot be produced by a combination of a thick disc and a thin disc with constant scale-heights and the scale-lengths because, then the global scale-height would be independent of $R$. If there is a thick disc with, for example, $h_{z}=910 \mathrm{pc}$, a density on the plane of $5.9 \%$ of the thin disc (Buser et al. 1999) and assuming that there is not significative difference in the abundances in the red clump population, then the thick disc would become dominant for $z>1300 \mathrm{pc}$ whereas for $z<500 \mathrm{pc}$ the thin disc would be by far the more important. Figure 8 shows very different slopes for different $R$ at $z<500 \mathrm{pc}$, and even the combination of thin and

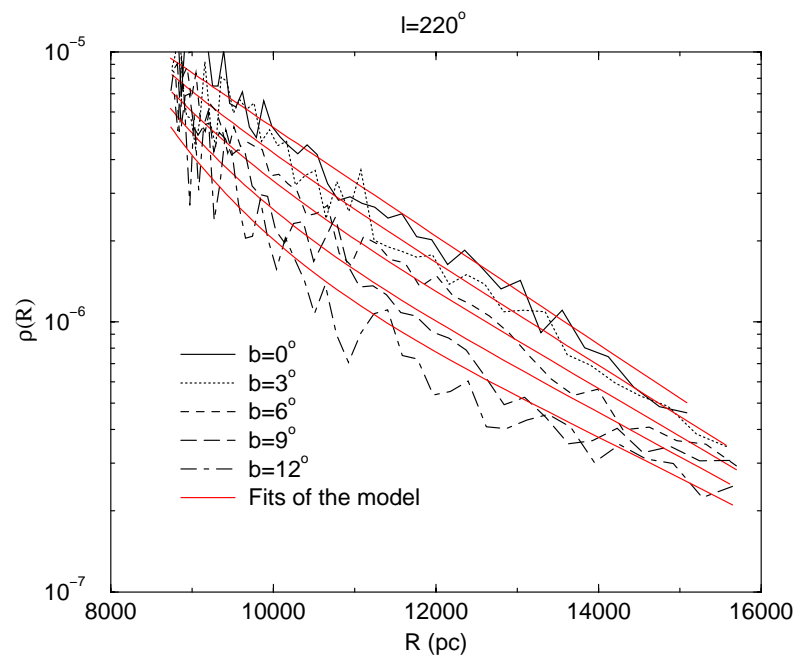

Fig. 9. The red clump star density obtained from the data and the lines showing a model (3) with $H=2.1 \mathrm{kpc}, h_{z}\left(R_{\odot}\right)=310 \mathrm{pc}, h_{R \text {,flare }}=$ $3.4 \mathrm{kpc}$ at $l=220^{\circ}$ and the latitudes stated on the plot.

thick disc with different scale-lengths cannot reproduce this effect because the thin disc is highly dominant for $z<500 \mathrm{pc}$. Furthermore, the thick disc cannot produce significant errors in the calculated scale-length, since this was derived in the plane where the thin disc always dominates. The scale-height, however, would be representative of an average of thin and thick discs. The average source height in the data set used is $\sim 300 \mathrm{pc}$, where the thin disc is $\sim 10$ times denser than the Buser thick disc.

Another noticeable feature of Figs. 7 and 9 is that the density follows the exponential to at least $15 \mathrm{kpc}$. As has already been discussed, if the disc had a sharp cut-off then this would cause an abrupt drop in the density. Hence the absence of such a sharp drop implies that there is no abrupt cut-off to the disc until at least $\sim 15 \mathrm{kpc}$. By $15 \mathrm{kpc}$ the star density has become too low to determine if there is a cut-off beyond that point.

\subsection{Flare in the inner Galaxy?}

The regions used in the previous subsection all had $|l|>90^{\circ}$, and hence the lines of sight run external to the solar circle $\left(R>R_{\odot}\right)$. Therefore, the results are valid only for the outer disc. Moreover, the flare scale-length of around $3.4 \mathrm{kpc}$ is really only a description of the flare for $R>10 \mathrm{kpc}$, as between 8 and $10 \mathrm{kpc}$ there is little data which is also very noisy (Fig. 7). Hence, whether this law is applicable to $R<10 \mathrm{kpc}$ is something that cannot be deduced from the previous analysis, and data with a range of lower galactocentric distances is required.

In order to examine the effect of the flare in the inner Galaxy this method should not be applied to lines of sight which cover only small a range of Galactocentric distances inside the solar circle, as these provide only a small range of values with which to attempt fitting $R$. For instance, on plane regions with $45^{\circ}<l<90^{\circ}$ would only give values of $R$ between 6 and $8 \mathrm{kpc}$. Therefore, in order to explore the inner disc using this method, we must use regions with $|l|<45^{\circ}$. Furthermore, the near plane regions with $|l|<30^{\circ}$ should also 


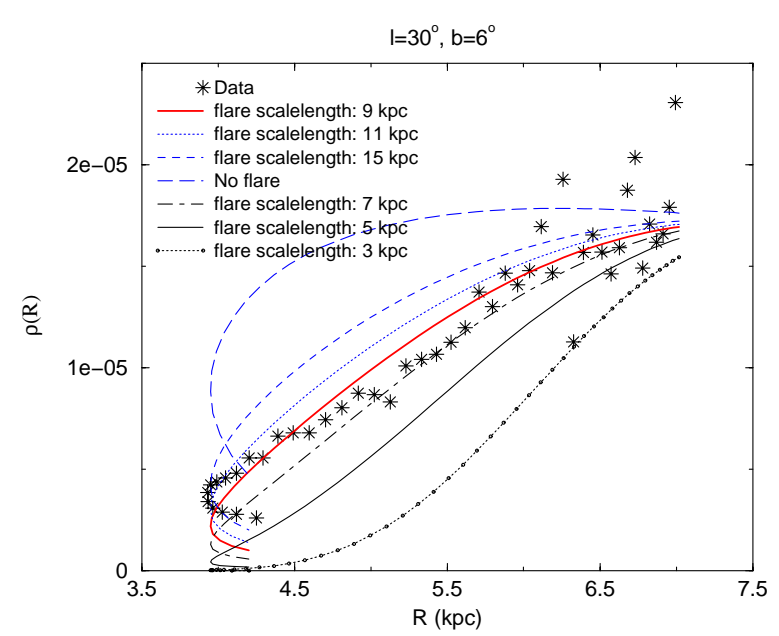

Fig. 10. The red clump star density obtained from the data and the lines showing a model (3) with $H=2.1 \mathrm{kpc}, h_{z}\left(R_{\odot}\right)=310 \mathrm{pc}$ and different flare scale-lengths ( $\left.h_{R, \text { flare }}\right)$ at $l=30^{\circ}, b=6^{\circ}$. As can be seen, a flare is necessary in the inner Galaxy, and its best scale-length is around $9 \mathrm{kpc}$.

be avoided, as the inner Galaxy components become important, e.g. the in-plane bar (Hammersley et al. 1994; LópezCorredoira et al. 2001). At present the available 2MASS data in the regions of interest is very restricted and so we will concentrate in only one region: $l=30^{\circ}, b=6^{\circ}$.

Figure 10 shows the density of the red clump giants along this line of sight determined using the same method as in Sect. 3.2. As well as the measured density, various model predictions are plotted. It shows that a flare is necessary to describe the data with a disc of $H=2.1 \mathrm{kpc}$ and $h_{z}\left(R_{\odot}\right)=310 \mathrm{pc}$. However, the scale-length of the flare cannot be as low as $3.4 \mathrm{kpc}$, but rather around $9 \mathrm{kpc}$. The scale-height in the inner Galaxy is smaller than the scale-height in the solar neighborhood, however the difference is only $h_{z}(5 \mathrm{kpc}) \approx 0.73 h_{z}\left(R_{\odot}\right)$. A flare scale-length of $3.4 \mathrm{kpc}$ would imply $h_{z}(5 \mathrm{kpc}) \approx$ $0.43 h_{z}\left(R_{\odot}\right)$. Hence, although the flare begins well inside the solar circle, the increase of the scale-height with $R$ is significantly slower in the inner Galaxy.

This result is consistent with previous work. For instance, Kent et al. (1991) has measured $h_{z}(5 \mathrm{kpc})=0.67 h_{z}\left(R_{\odot}\right)$ by fitting the parameters of the disc in a $2.4 \mu \mathrm{m}$ map of the northern Galactic plane.

\section{Using the star counts to obtain the parameters of the disc}

The second method used for determining the parameters for the stellar density is by the fitting of star counts in different regions. For each of the line of sight centered on Galactic coordinates $(l, b)_{i},\left(i\right.$ is the field number) the cumulative star counts $\left(N_{K}\right)$ observed in $K$, up to a magnitude $m_{K}$ in a given solid angle $\omega$ is the sum of the stars within the beam brighter than the given apparent magnitude (Bahcall 1986). Assuming that the luminosity function does not vary with the spatial position, this is

$$
\begin{aligned}
& N_{K}\left(m_{K}, l, b\right)=\omega \\
& \times \int_{0}^{\infty} \Phi_{K}\left(M_{K}(r, l, b)\right) \rho(R(r, l, b), z(r, l, b)) r^{2} \mathrm{~d} r,
\end{aligned}
$$

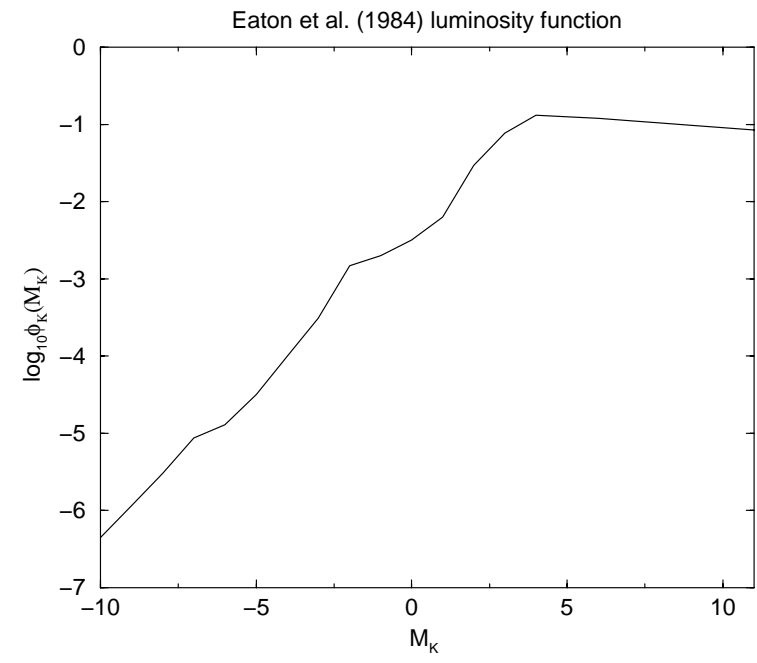

Fig. 11. $K$-band normalized luminosity function used in the second method of this paper, from Eaton et al. (1984).

where

$M_{K}(r, l, b)=m_{K}+5-5 \log _{10} r-A_{K}(r, l, b)$,

$\Phi_{K}\left(M_{K}\right)=\int_{-\infty}^{M_{K}} \phi_{K}(M) \mathrm{d} M$,

$\phi_{K}$ is the normalized luminosity function for the $K$ band in the disc and $A_{K}(r, l, b)$ is the $K$ extinction along the line of sight $(l, b)$ up to distance $r$. We have used a standard disc luminosity function given in Eaton et al. (1984, see Fig. 11) and the extinction model given by Wainscoat et al. (1992) (except with $h_{R \text {,dust }}=3.2 \mathrm{kpc}$ to scale the distance to the center of the Galaxy to $7.9 \mathrm{kpc}$ ). Fitting could also be used to constrain the extinction, however it is preferable to have as few free parameters as possible. The extinction model of Wainscoat et al. (1992) is axisymmetric and does not include a flare or a warp in the dust distribution. However, $K$-band counts are not very sensitive to small variations of the extinction and were extinction the goal of the paper shorter wavelengths would be more appropriate. Therefore, as the lines of sight are in general looking towards the outer Galaxy and the extinction is low, a simple extinction model will suffice.

The stellar density $(\rho)$ parameters (Eq. (3)) were determined using the available 2MASS data at $b=0, \pm 3, \pm 6, \pm 9$ degrees; $45^{\circ}<l<315^{\circ}$. On or near-plane regions were preferred because these are more sensitive to the variations in the parameters, and there are significantly more stars. Again the central regions of the Galaxy were avoided so that other components other than the disc would not be included. It should be noted that even when the local stars dominate the counts there are still sufficient giants to constrain the large scale structure. Each region used an area of 1 square degree $\left(\Delta l=1^{\circ}\right.$, $\Delta b=1^{\circ}$ ). However, some areas are only partially covered and in these cases the counts were divided by the actual area available to obtain the counts per unit area. In total 820 lines of sight with areas between 0.5 and $1.0 \mathrm{deg}^{2}$ are used. This gives a very large area coverage along the Galactic plane, and so can constrain the parameters of the disc. Other authors have used 


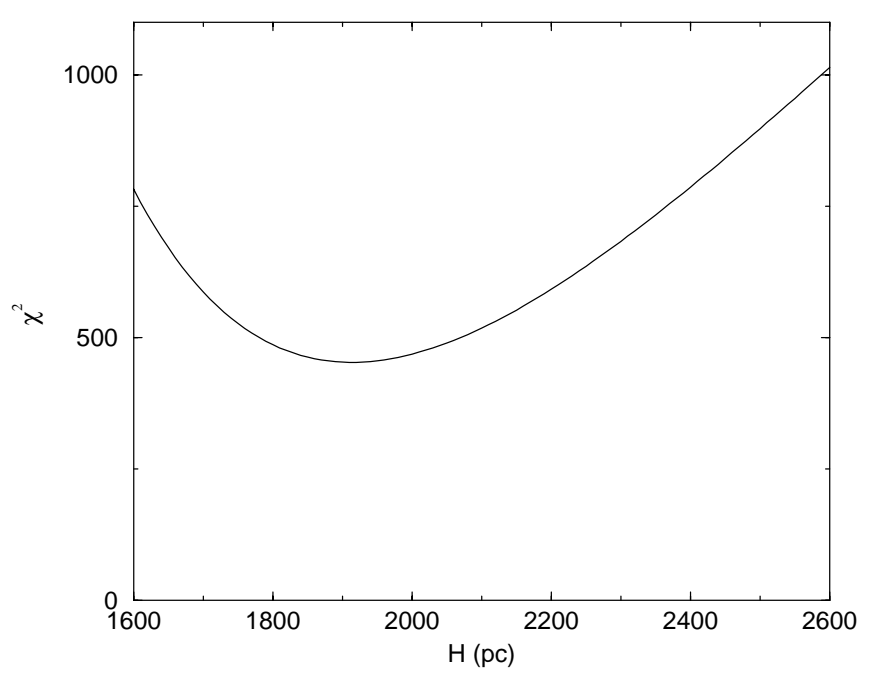

Fig. 12. $\chi^{2}$ of the fit of star counts in the plane for the model (6).

2MASS star counts to study the disc however the number of regions used is much lower. Ojha (2001) used seven independent regions quite far from the plane but does not study the warp, flare and cut-off whilst Alard (2000) only looked at three strips in latitude across the plane.

As noted, this method assumes that all sources have the same spatial distribution, i.e. the scale-height and scale-lengths in a given galactocentric radius are the same. For the scaleheight, in particular, this is known not to be true with the younger components having a scale-height far less than the older components. However, the young components only make a significant contribution to the counts at the brightest magnitudes $\left(m_{K}<8\right)$ and even then only on the plane towards the inner Galaxy (Hammersley et al. 1999). For this study we have used 2MASS counts up to $m_{K}=14.0$. This magnitude limit, coupled with using lines of sight away from the inner Galaxy, means that the counts will be completely dominated by the old population of the disc rather than the young disc and spiral arms. Hence the assumption that all of the sources will have, at least, very similar scale-heights is justified.

We have limited the maximum apparent magnitude in $K$ band to 14.0. This is well below the limiting magnitude of 2MASS and ensures that there are complete statistics in all regions. Moreover, with this limiting magnitude, stars brighter than $M_{K}=0$ can be detected to over $6 \mathrm{kpc}$ which means the results are sensitive to the shape of the very outer disc. However, one of the curious features of stars counts is that the fainter the magnitude the closer on average are the sources being detected. This is caused by the steeply rising luminosity function and means that the dwarfs will dominate the fainter counts. The result is that the red clump giant method, which isolates a specific group of stars, will provide a better contrast for the more external part of the disc.

As discussed previously, the "old disc" analyzed here includes all the old stars of the disc within the selected regions (average latitude 4-5 degrees). If there is a thick disc like the one proposed by Buser et al. (1999), the mean contribution to the star counts in the selected regions would be around $10 \%$,

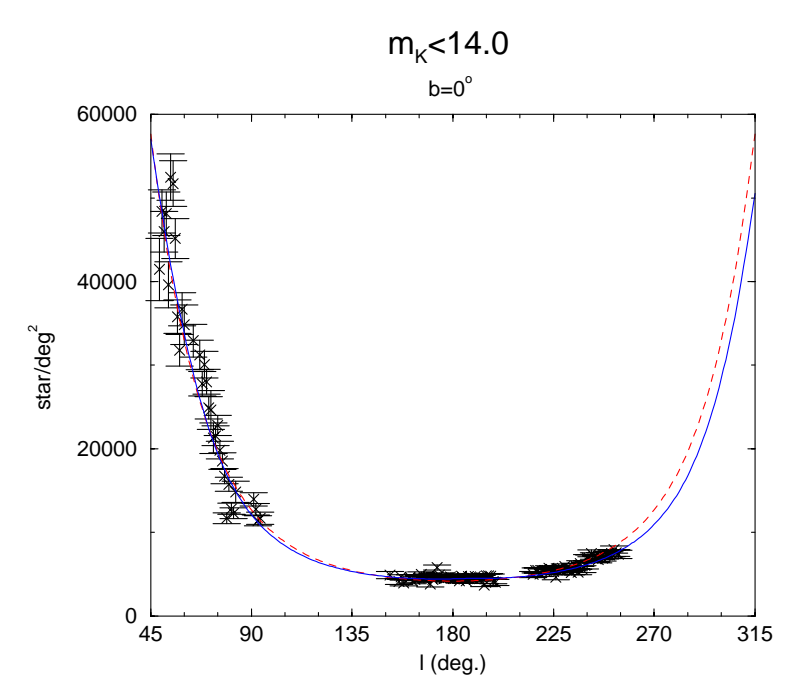

Fig. 13. Fit of star counts in the plane. The dashed line is the best nonwarped model $(H=1.91 \mathrm{kpc})$. Solid line is the best warped model $\left(H=1.97 \mathrm{kpc} ; \epsilon_{\mathrm{w}}=5.25, \phi_{\mathrm{w}}=-5^{\circ}, A_{\mathrm{w}}=2.1 \times 10^{-19} \mathrm{pc}\right)$.

assuming a thick disc luminosity function similar to that of the halo in Wainscoat et al. (1992). Hence, the scale-height determined here would be representative of an average of the thin and thick discs but with $90 \%$ of the sources coming from thin disc.

Star counts cannot easily distinguish between the cut-off and the loss of stars in the plane due to the flare. However, it was shown in the previous section that it there is no cut-off to at least $15 \mathrm{kpc}$, and so we will continue to assume there is not a cut-off.

\subsection{Results}

As in Sect. 3, the fitting of the parameters will be in two steps. The first step is to fit $H$ using the on plane regions $(b=0)$ and then fit $h_{z}\left(R_{\odot}\right)$ and $h_{R \text {,flare }}$ using all of the regions.

Using the available regions in the plane the minimum $\chi^{2}$ $\left(\chi^{2}=452, N=114\right.$ ) was obtained for (see Fig. 12):

$H=1.91_{-0.16}^{+0.20} \mathrm{kpc}$.

The stellar density in the solar neighborhood is $\rho_{\odot}=5.3 \times$ $10^{-2} \mathrm{star}_{\mathrm{pc}}^{-3}$. This is somewhat lower than the value measured in other surveys (e.g., $0.13 \mathrm{star} / \mathrm{pc}^{3}$, Bahcall 1986). This discrepancy is most probably due to using different luminosity functions, here we used the normalized luminosity function by Eaton et al. (1984). In any case the normalization does not affect the other disc parameters. The best fit is shown in the Fig. 13 (dashed line).

The other two parameters were then fitted using all the available data at $45^{\circ}<l<315^{\circ}$ and $b=0^{\circ}, \pm 3^{\circ}, \pm 6^{\circ}, \pm 9^{\circ}$. The minimum $\chi^{2}$ is found for

$$
\begin{aligned}
& h_{z}\left(R_{\odot}\right)=300_{-15}^{+13} \mathrm{pc}, \\
& h_{R, \text { flare }}=4.6 \pm 0.5 \mathrm{kpc} .
\end{aligned}
$$



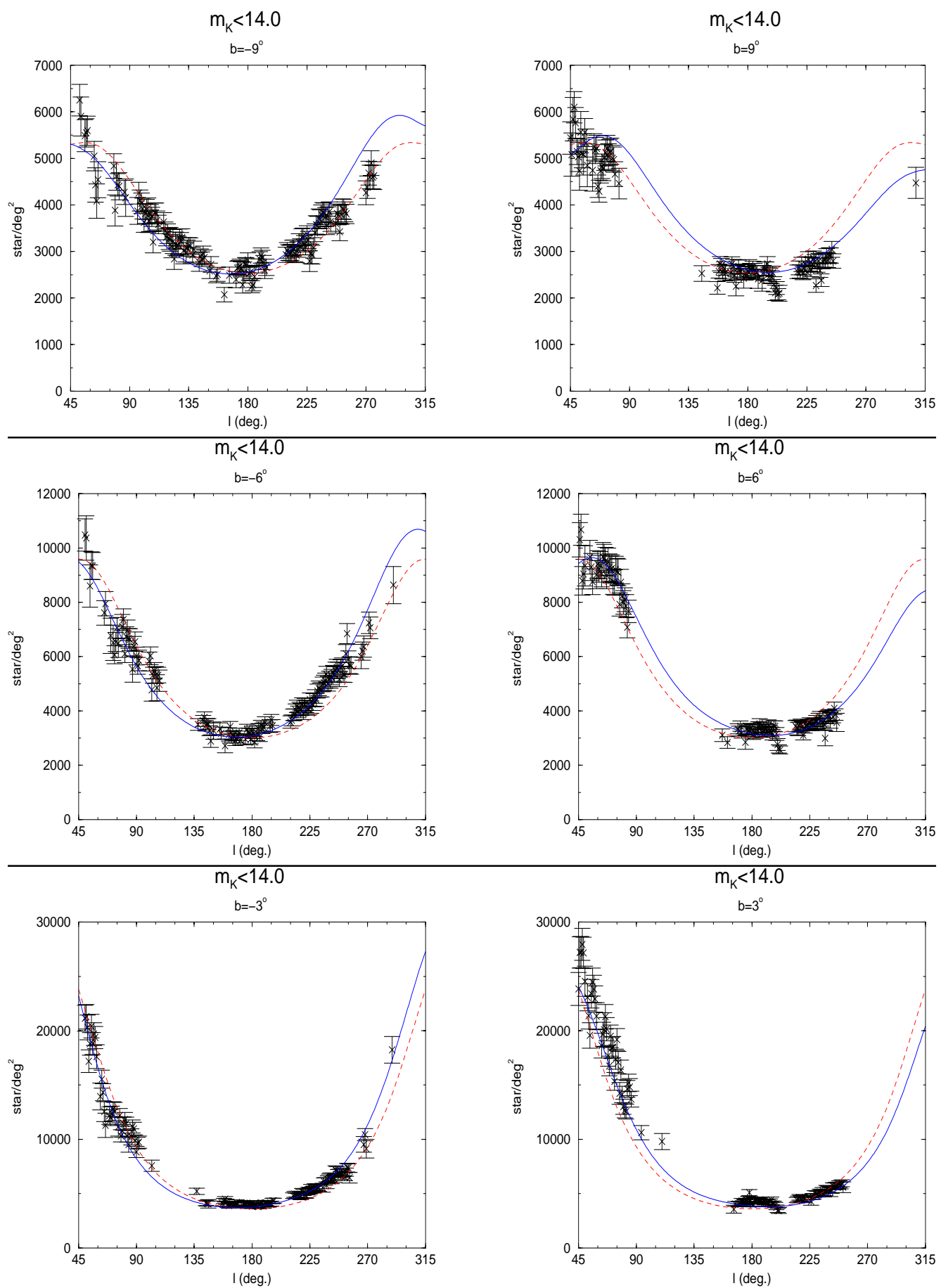

Fig. 14. Data and fit of star counts. The dashed line is the best non-warped model $\left(H=1.91 \mathrm{kpc}, h_{z}\left(R_{\odot}\right)=300 \mathrm{pc}, h_{R \text {,flare }}=4.60 \mathrm{kpc}\right)$. The solid line is the best warped model $\left(H=1.97 \mathrm{kpc}, h_{z}\left(R_{\odot}\right)=285 \mathrm{pc}, h_{R, \text { flare }}=5.00 \mathrm{kpc} ; \epsilon_{\mathrm{w}}=5.25, \phi_{\mathrm{w}}=-5^{\circ}, C_{\mathrm{w}}=2.1 \times 10^{-19} \mathrm{pc}\right)$.

These errors are for $H=1.91 \mathrm{kpc}$ and other values of $H$ could slightly change the error ranges. In this case, the best fit was for $\chi^{2}=3233$ with $N=820$. The fits are shown in Figs. 13 and 14 (dashed line).

In general there is a good fit, although there are some asymmetries between $45^{\circ}<l<180^{\circ}$ and $180^{\circ}<l<315^{\circ}$ and also between $b>0$ and $b<0$. These can be attributed principally to the warp (see Sect. 5), but there are also some other minor fluctuations, possibly due to the patchiness of the extinction or contamination from other Galactic components.

\section{In search of the stellar warp}

If there were neither a warp nor other asymmetries, the ratio of counts above and below the plane (Fig. 15) should be nearly the same for all Galactic longitudes, with only a small offset due to the height of the Sun above the plane. However, the plot clearly show a sinusoidal behavior. There is an excess of counts at positive latitudes with respect the negatives for $l<180^{\circ}$ and the opposite for $l>180^{\circ}$. A warp, together with a small offset due the height of the Sun above the plane, can explain what is seen. 

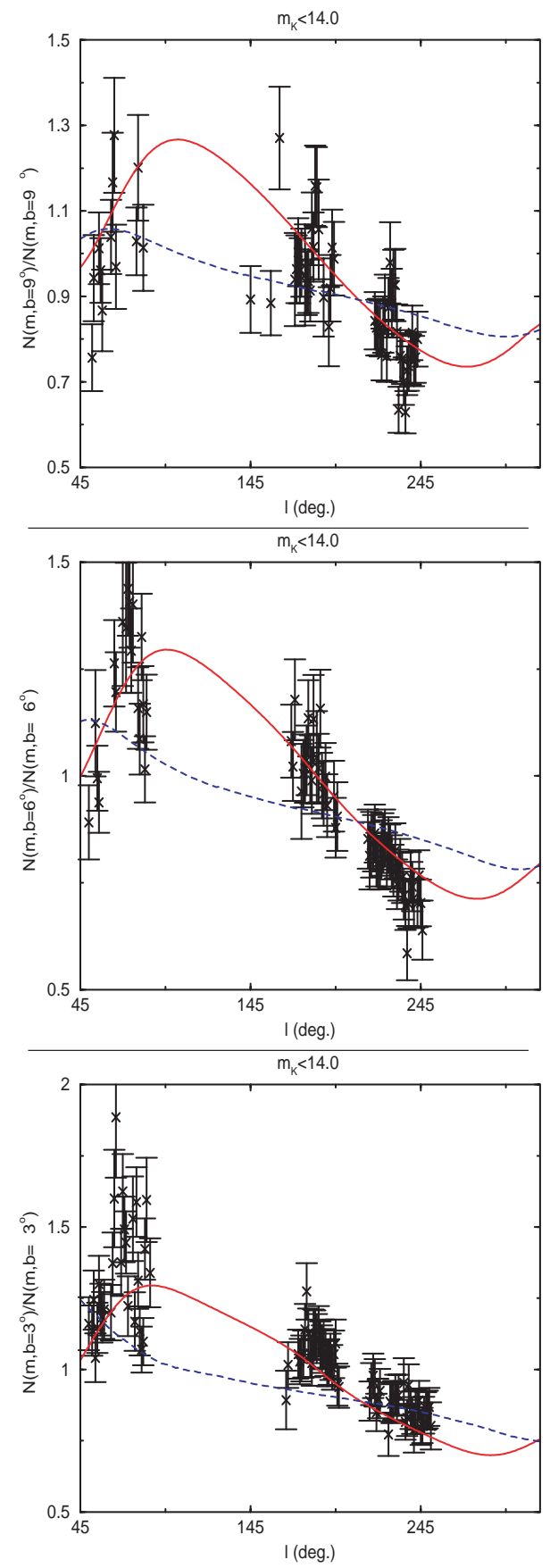

Fig. 15. Positive counts divided by the negative counts. The solid line stands for a warped model with $H=1.91 \mathrm{kpc}, h_{z}\left(R_{\odot}\right)=300 \mathrm{pc}$, $h_{R, \text { flare }}=4.60 \mathrm{kpc} ; \epsilon_{\mathrm{w}}=5.25, \phi_{\mathrm{w}}=-5^{\circ}, A_{\mathrm{w}}=2.1 \times 10^{-19} \mathrm{pc}$. The dashed line stands for the best "tilted disc" model with $H=1.91 \mathrm{kpc}$, $h_{z}\left(R_{\odot}\right)=300 \mathrm{pc}, h_{R, \text { flare }}=4.60 \mathrm{kpc} ; b_{\text {corrected }}=b+1.64^{\circ} \sin \left(l+170^{\circ}\right)$.

A model similar to Eq. (3) will be used, but with $\left|z-z_{\mathrm{w}}\right|$ instead of $|z|$, where $z_{\mathrm{W}}$ is the average elevation of the disc above a plane which is parallel with $b=0$ but runs though the local center of mass (i.e. 15 pc below the Sun).

$z_{\mathrm{w}}=\left[C_{\mathrm{w}} R(\mathrm{pc})^{\epsilon_{\mathrm{w}}} \sin \left(\phi-\phi_{\mathrm{w}}\right)+15\right] \mathrm{pc}$.

Hence, the model describes the warp as a series of tilted rings, where the amount of tilt is the galactocentric distance raised to a power $\epsilon_{\mathrm{w}}$. The $15 \mathrm{pc}$ term is due to the height of the Sun above the plane (Hammersley et al. 1995). Since the value of about

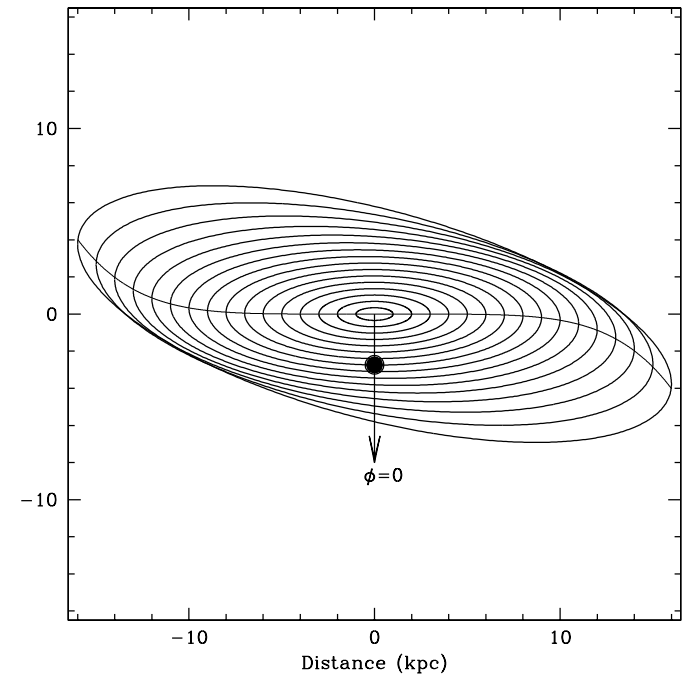

Fig. 16. A schematic of the warp showing the relative location of the Sun. The rings are each kpc, and the line of the maximum warp is shown.

$15 \mathrm{pc}$ is well constrained by other studies we will not carry out an analysis of this parameter. The two warp parameters, $\epsilon_{\mathrm{w}}$, and the galactocentric angle $\phi_{\mathrm{w}}\left(\phi_{\mathrm{w} \odot}=0\right)$, are determined from the division of star counts data in Fig. 15. Once the parameters are fixed then $C_{\mathrm{w}}$ is obtained from:

$C_{\mathrm{w}}=\frac{\sum_{i} \frac{d_{i} t_{i}}{\sigma_{i}^{2}}}{\sum_{i} \frac{t_{i}^{2}}{\sigma_{i}^{2}}}$,

where $d_{i}=\left(\frac{N(m,+b)}{N(m,-b)}-1\right)$ with $N(m, b)$ the observational cumulative counts, $\sigma_{i}$ the errors of $d_{i}$, and $t_{i}$ is the model prediction for $d_{i}$ with $C_{\mathrm{w}}=1$.

In this way, there are only two free parameters in the model and so it is simpler to constrain the warp. The model assumes that the northern and southern warp are symmetric, apart from the effect of the height of the Sun above the plane. This is an approximation and it is known that the gas warp is not symmetric (Burton 1988). However, the asymmetries of the warp are produced over galactocentric distances $R \approx 13 \mathrm{kpc}$ (distance from the Sun: $r>8 \mathrm{kpc}$ for $l=250^{\circ}$ ), where there are few stars from the total sample of stars with $m_{K}<14.0$.

\subsection{Results}

The best fit is found for:

$\epsilon_{\mathrm{w}}=5.25 \pm 0.5$

$\phi_{\mathrm{w}}=-5 \pm 5$ degrees,

which gives $C_{\mathrm{w}}=2.1 \times 10^{-19} \mathrm{pc}$ with $\chi^{2}=332.7, N=217$. The best fit is shown in Fig. 15 (solid line). Although there are few points which do not fit very well (perhaps due to irregularities in the extinction or in the distribution, contamination of the spiral arms, etc.) again the general fit is good. Hence, a warp does accurately describe the deviations from the best symmetric disc. In Fig. 16, a representation of the warp is plotted. 


\subsection{Could this be a local tilted disc effect?}

An alternative hypothesis that could explain the asymmetries is that the disc is locally tilted with respect to the global galactic plane. Hammersley et al. (1995) showed that a similar asymmetry COBE-DIRBE surface brightness-maps could be caused by a local tilt to the disc. However, Hammersley et al. (1995) deliberately chose regions well away from the plane, so that the results would be dominated by local sources and there would be little contribution from regions where the warp becomes significant $(R>11 \mathrm{kpc})$. Star count data provide direct information on the distance to the sources, something not available with surface brightness-maps and so can better distinguish between a tilt and warp.

In Fig. 15, it is shown the best fit for a tilted disc model, i.e. assuming that the asymmetries are only due to the height of the Sun above the plane and the local tilt of the disc plane, which corrects the Galactic latitude as (Hammersley et al. 1995):

$z_{\mathrm{w}}=15 \mathrm{pc}$,

$b_{\text {corrected }}=b+\alpha_{\mathrm{t}} \sin \left(l+l_{\mathrm{t}}\right)$.

The best fit for the tilt corresponds to

$l_{\mathrm{t}}=170 \pm 20 \mathrm{deg}$,

which gives a value of $\alpha_{\mathrm{t}}=1.64^{\circ}$ and $\chi^{2}=517(N=217)$. The orientation found is in good agreement with Hammersley et al. (1995), but the tilt is some 4 times greater indicating sources at a significantly larger distance are being detected.

The predictions for the tilt are significantly poorer than for the model of the warp, as can be seen in Fig. 15 (dashed line). Hence, whilst a small tilt is sufficient to describe the geometry of local disc, beyond a few kpc this simplification is no longer valid. Interestingly the average tilt predicted by the warp model over the distance range used in Hammersley et al. (1995) (i.e. about $2 \mathrm{kpc}$ ) is still about twice as large as that measured from the surface brightness map. This suggests that the warp actually starts just outside the solar circle, although it should be noted that the predicted effect in the solar vicinity is small in any case and the star counts are not sensitive for very short distances from the Sun.

\subsection{Confirmation of the warp with the red clump giants}

A warp should also leave a clear imprint in the distribution of the red clump giants (Sect. 3). Since the warp will affect the distant sources most, the asymmetries should be greater at larger galactocentric distances. This can be seen in Fig. 17, where the ratio of densities at positive and negative latitude is shown for the region $l=220^{\circ}, b= \pm 6^{\circ}$. The data show a strong asymmetry with most points having a ratio $<0.8$ whilst for those beyond $13 \mathrm{kpc}$ it drops to under 0.6. This again shows that the asymmetries in the counts are larger for distant sources. To confirm that asymmetries cannot be due to the height of the Sun above the plane, the dashed line in Fig. 17 shows the predicted level of this effect. The predicted warp, however, is shown as the solid line and this does provide a reasonable fit.

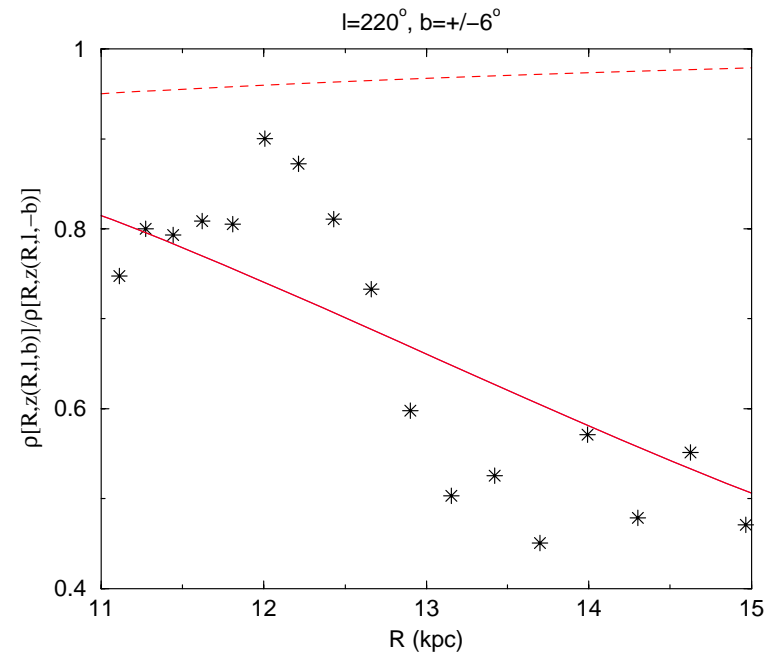

Fig. 17. Ratio of densities between positive and negative latitude for ( $l=220^{\circ}, b= \pm 6^{\circ}$ ). The solid line shows the predicted ratio for the warp model with $H=1.91 \mathrm{kpc}, h_{z}\left(R_{\odot}\right)=300 \mathrm{pc}, h_{R \text {,flare }}=4.60 \mathrm{kpc}$; $\epsilon_{\mathrm{w}}=5.25, \phi_{\mathrm{w}}=-5^{\circ}, C_{\mathrm{w}}=2.1 \times 10^{-19} \mathrm{pc}$. The dashed line shows the effect of the height of the Sun above the plane $(+15 \mathrm{pc})$.

Another aspect which needs to be commented upon is that the measured flare scale-length may be affected by the warp, even in regions where the warp is not very conspicuous $\left(l=220^{\circ}\right)$. Figure 17 shows that the ratio may be as low as 0.6 , i.e. an error of around $20 \%$ for each of the densities at positive and negative latitude. This is one reason why the results from (14) should be taken with some care. However, in the second method, the relative contribution from the very distant stars $(R>\sim 12 \mathrm{kpc})$ is much lower and the effect of the warp is smaller (compare the results between Sects. 4.1 and 5.5). Hence, the parameters derived from the star counts are probably more trustworthy than those derived from the red clump giants in Sect. 3.

\subsection{Coincidence of stellar and gas warp}

The measurements of stellar warp presented here are totally independent of those used to determine gas warp (Burton 1988; Diplas \& Savage 1991). It is noticeable however, that the stellar warp is nearly identical to that of the gas. Not only is the angle of orientation of the maximum amplitude $\left(\phi \approx 85^{\circ}\right)$ coincident, but the amplitude as well (see Fig. 18). The northern warp in both the gas and stars are very similar to $R>13 \mathrm{kpc}$, as shown in Fig. 18. The southern gas warp differs from the northern gas warp, becoming constant or decreasing with height for $R>$ $13 \mathrm{kpc}$. However, since a large majority of the stars along the line of sight of southern warp $\left(l \approx 250^{\circ}\right)$ have $R<13 \mathrm{kpc}$ $(r<8 \mathrm{kpc})$, the data are not sensitive enough to detect the differences between the northern and southern warp.

Other authors have also looked for the stellar warp. Djorgovski \& Sosin (1989) obtained a similar result with IRAS sources. Alard (2000) also measured the stellar warp using 2MASS data, and also concluded that the amplitude is roughly coincident with the gas warp. Although the present results are more accurate, Alard (2000) showed that the amplitude of the stellar warp is consistent with the HI to within 


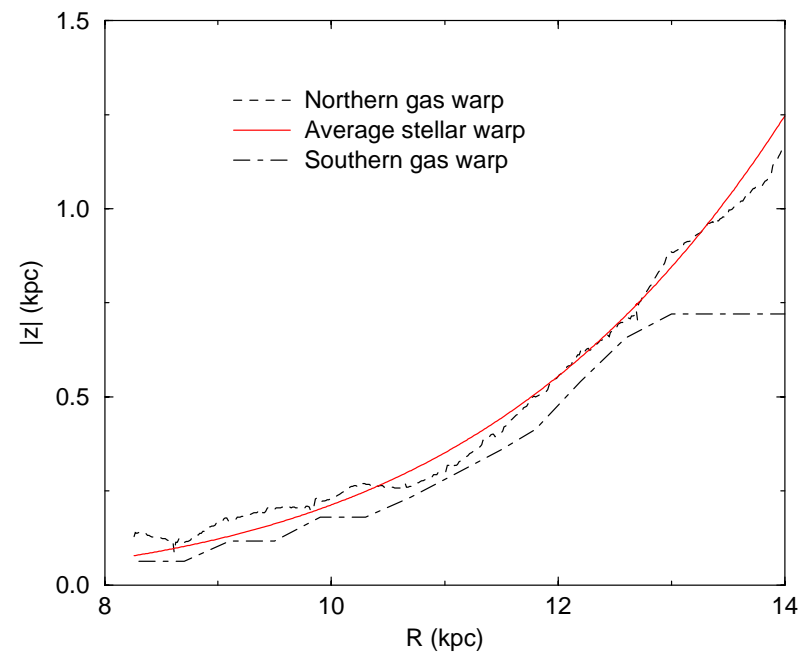

Fig. 18. Maximum amplitude of the stellar warp (solid line: $z_{w}=$ $2.1 \times 10^{-19} R(\mathrm{pc})^{5.25} \mathrm{pc}$, which is the best power-law fit to the data) in comparison with the one measured by Burton (1988) for the northern and southern warp gas scaled to $R_{\odot}=7.9 \mathrm{kpc}$ (dashed and dot-dashed lines).

about 30\%. Drimmel \& Spergel (2001) found from COBEDIRBE data that the stellar warp is more or less coincident with the stellar warp found here (and Burton 1988 gas warp): $z_{\mathrm{w}} \approx 25 \mathrm{pc} / \mathrm{kpc}^{2}(R-7 \mathrm{kpc})^{2}$ and Freudenreich (1998) obtained similar results in his independent determination of warp parameters.

From the presented results, it is clear that the form of the stellar warp is identical to that of the gas warp. Hence, whatever is causing of the warp affects both to the stars (including the old population) and gas in approximately the same way. Whilst the stellar and gas warps are coincident for the Milky way, this may not necessarily be the case in other galaxies.

\subsection{A new fit of the disc including the warp}

After obtaining the parameters of the warp, which is a second order effect on the total counts, it is possible update the disc model to include the warp and then redetermine the parameters using the cumulative star counts. As expected, there are no major changes with respect to the previous values (18), (19):

$$
\begin{aligned}
& H=1.97_{-0.12}^{+0.15} \mathrm{kpc} \\
& h_{R, \text { flare }}=5.0 \pm 0.5 \mathrm{kpc} \\
& h_{z}\left(R_{\odot}\right)=285_{-12}^{+8} \mathrm{pc} .
\end{aligned}
$$

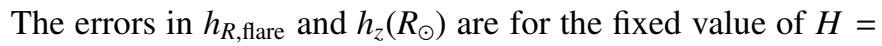
$1.97 \mathrm{kpc}$. Other values of $H$ could change very slightly the error ranges. This best fit gives $\rho_{\odot}=5.5 \times 10^{-2}$ and corresponds to $\chi^{2}=352$ for the data in the plane $(N=114)$, and $\chi^{2}=2049$ for the whole sample $(N=820)$. The fits are plotted in Figs. 13 and 14 (solid line). Using Eq. (4), this provides a constraint on the intrinsic scale-length of the disc:

$h_{R}=3.3_{-0.4}^{+0.5} \mathrm{kpc}$.
It should be remembered that these parameters have between determined between about $R=6$ and $R=15 \mathrm{kpc}$ (between $0.75 R_{\odot}$ and $1.9 R_{\odot}$ ). No data comes from $R<6 \mathrm{kpc}$ as the limit in longitude is $45^{\circ}$ and beyond $15 \mathrm{kpc}$ the data becomes uncertain.

\section{Comparison of both methods for the flare and discussion}

In Sect. 3, red clump giants gives values compatible with those

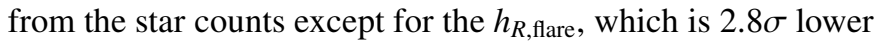
$(3.4 \mathrm{kpc}$ instead of $5.0 \mathrm{kpc})$. It is unlikely that this is a separate population with different flare, because the scale-height at the solar circle is coincident. We suspect the difference stems from a selection effect. Apart from the fact the measurement of red clump giants is affected by the warp and hence the value of 3.4 is somewhat less trustworthy (Sect. 5.3), it must be remembered that most of the giants are at $R>12 \mathrm{kpc}$, i.e. are dominated by distant stars. However, the flare scalelength is not constant, it is higher for smaller $R$ (Sect. 3.6 shows that $h_{R \text {,flare }} \approx 9 \mathrm{kpc}$ between $R=4 \mathrm{kpc}$ and $R=7 \mathrm{kpc}$ ). Hence, a systematic difference between the two values should be expected.

The flare scale-length can be expressed as:

$h_{R, \mathrm{flare}} \approx 12-0.6 R \mathrm{kpc}$,

where $R$ is in $\mathrm{kpc}$. This gives the values of $9.0 \mathrm{kpc}$ for $R=5 \mathrm{kpc}$ (close to the average galactocentric distance of the sources along $l=30^{\circ}, b=6^{\circ}$ ); $5.0 \mathrm{kpc}$ for $R=11.7 \mathrm{kpc}$ (representative of the range between 6 and $15 \mathrm{kpc}$ ); and $3.4 \mathrm{kpc}$ for $R=14.3 \mathrm{kpc}$ (representative of the range occupied by the distant giants). In the solar neighborhood, the value of $h_{R \text {,flare }}$ would be $7.3 \mathrm{kpc}$.

In comparison, Alard (2000) has described the flare in 2MASS data by $h_{z}=h_{z}\left(R_{\odot}\right)\left(1+\left(0.32 \mathrm{kpc}^{-1}\right)\left(R-R_{\odot}\right)\right)$. This is similar to our results in the range $8<R<12 \mathrm{kpc}$. Hence these results are in agreement with Alard (2000) and the thickening of the disc explains the rapid drop of the density in the outer Galaxy without the need to invoke a cut-off for $R<15 \mathrm{kpc}$. Indeed, as we have seen in Sect. 3, the cut-off at $R<15 \mathrm{kpc}$ is not only unnecessary but inconsistent with the data.

The flare, like the warp, is another characteristic of the gas in the outer Galaxy. Again the parameters determined for the stars are very similar to that of the gas (Wouterloot et al. 1990), although the gas scale-height is always far lower.

\subsection{Application of the disc model to brighter apparent magnitudes}

An aspect which must be examined is the possible variation of the measured local stellar density $\rho_{\odot}$ when using a range of magnitudes other than $m_{K}<14.0$. In principle, the given density distribution should be independent of the magnitude range used, however, there may be errors in Eaton's normalized luminosity function that has been used here. Alternatively there could be source confusion at the faintest 2MASS magnitudes, although such an effect would at most only affect the 


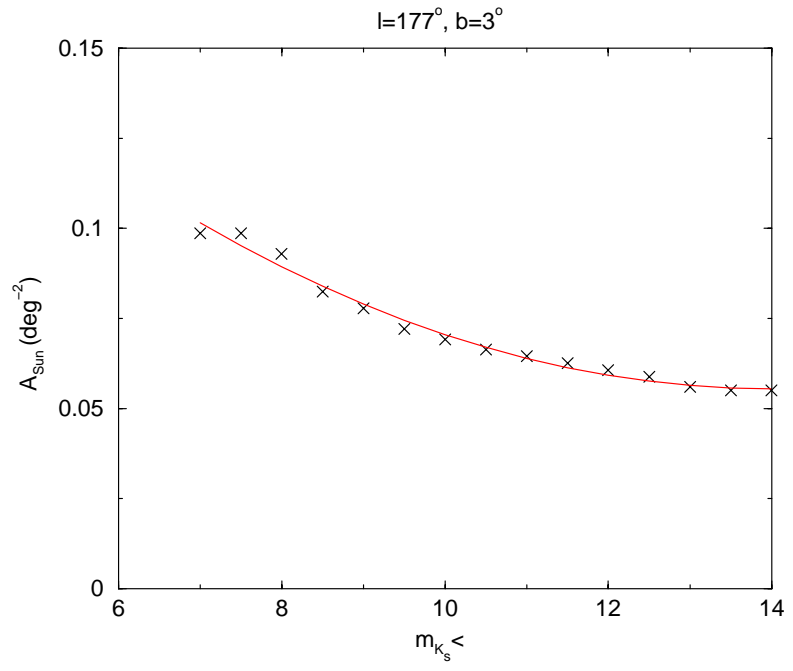

Fig. 19. Variation of the amplitude $\rho_{\odot}$ as a function of the maximum $K$-band magnitude when we use the disc model of this paper together with Eaton et al. (1984) luminosity function. Solid line is Eq. (28).

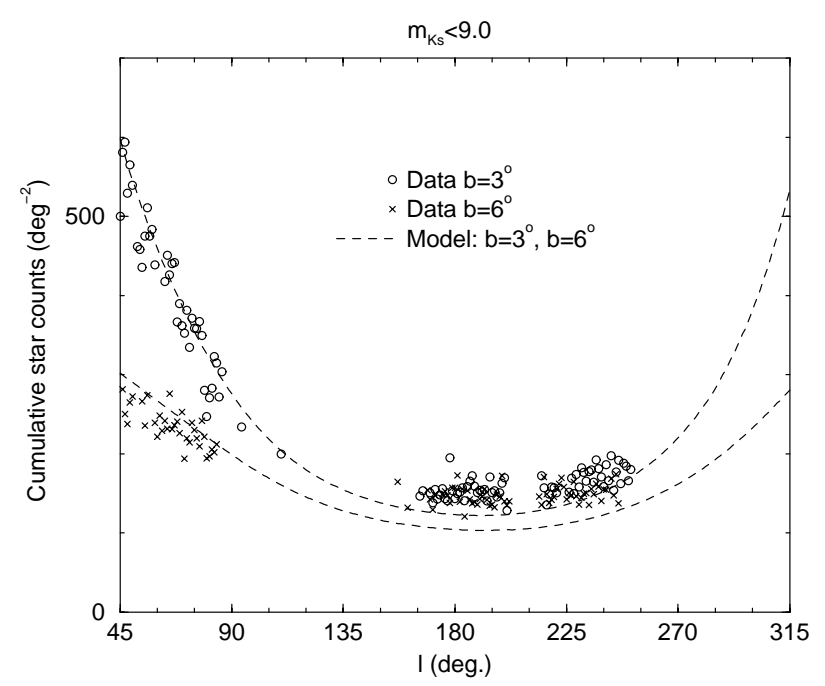

Fig. 20. Data and fit of star counts for $m_{K}<9.0$. The dashed line is the best warped model $\left(H=1.97 \mathrm{kpc}, h_{z}\left(R_{\odot}\right)=285 \mathrm{pc}, h_{R, \text { flare }}=5.00 \mathrm{kpc}\right.$; $\left.\epsilon_{\mathrm{w}}=5.25, \phi_{\mathrm{w}}=-5^{\circ}, A_{\mathrm{w}}=2.1 \times 10^{-19} \mathrm{pc}\right)$.

sources within a magnitude of the survey limit in the regions measured. Both errors would affect the measured density, $\rho_{\odot}$. Figure 19 shows the variation of $\rho_{\odot}$ with limiting magnitude $m_{K}<m_{K, \text { max }}$. This can be described as

$$
\begin{aligned}
\rho_{\odot} \approx & 0.2402-2.642 \times 10^{-2} m_{K, \text { max }} \\
& +9.446 \times 10^{-4} m_{K, \text { max }}^{2} .
\end{aligned}
$$

The data were obtained by normalizing the star counts with a circular region centered in $\left(l=177^{\circ}, b=3^{\circ}\right)$ with radius $=$ 2 degrees. The form of variation is not consistent with there being confusion and so it has to be due to errors in the form of Eaton's luminosity function.

An alternative possibility for this variation could have been the density parameters are not correct and for this reason $\rho_{\odot}$ is not constant with $m_{K}$. This can be checked by fitting of the star counts with the model described in Sect. 5.5 but changing the limiting magnitude and hence $\rho_{\odot}$ using Eq. (28). It is found that
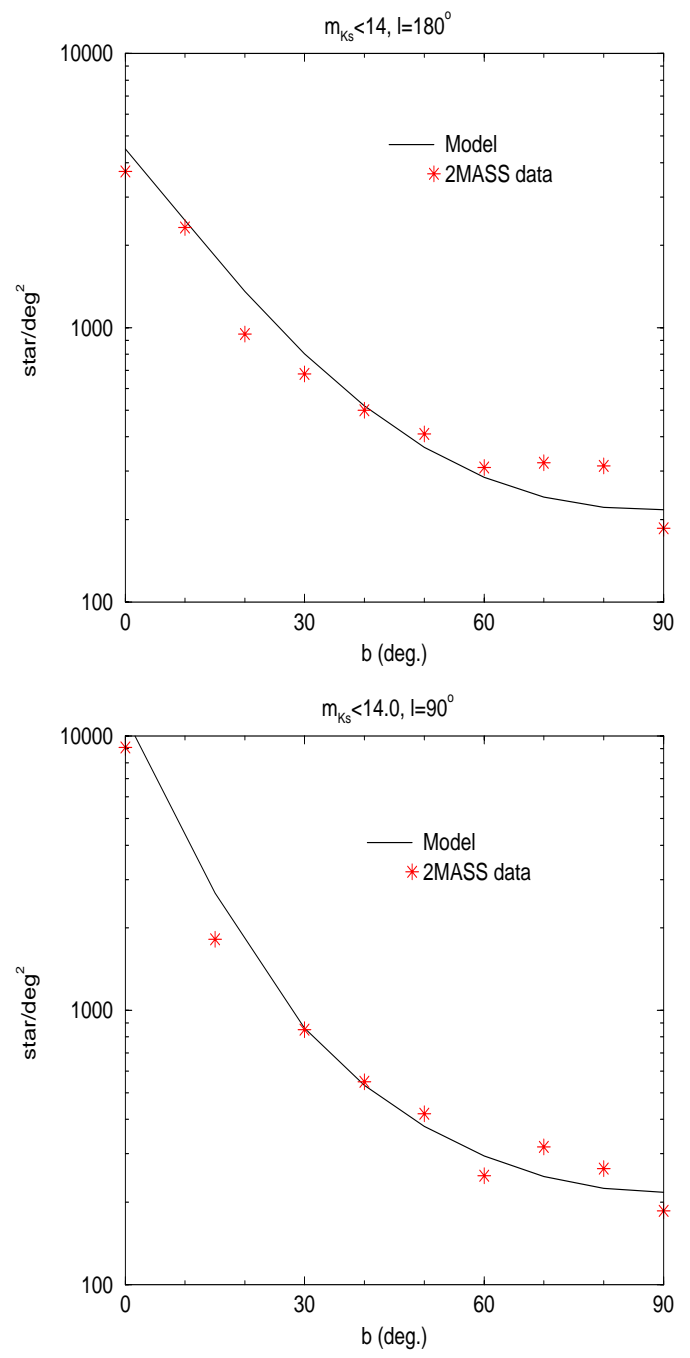

Fig. 21. Star counts in high latitude regions. Note how the model derived in the present paper (fitting low latitude regions) also fit approximately these high latitude regions. Data come from the available covered regions in the different coordinates within a radius of 2 degrees.

the model still predicts the measured star counts (some excess in the anticenter could be due to some contaminant of bright sources). As an example Fig. 20 shows the fit for the whole plane for $m_{K}<9.0$.

\subsection{Application to high latitudes}

Figure 21 shows how well the model fits the counts at high latitude regions. Even near polar regions $\left(b=90^{\circ}\right)$ the model predictions do not systematically deviate from the counts. It should be noted that the model does not include a bimodal distribution, containing both a thin and thick disc with different populations and scale-heights. None the less using the thin disc alone the model gives an accurate prediction of the counts, for the magnitude range being used. It is beyond the scope of this paper to discuss whether this result should be interpreted as an absence of thick disc or whether it is simply that the analysis presented here is not sensitive to the thick disc. A more detailed 


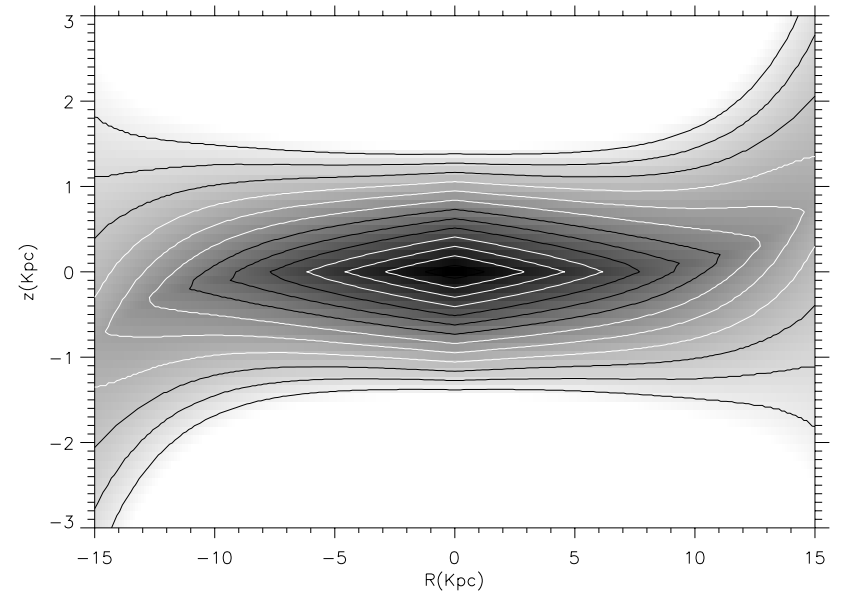

Fig. 22. Cut in the plane $Y Z$ of the Galaxy as described by the final disc model.

examination of high galactic latitudes, including an analysis of the colors and luminosity function, would be required.

\section{Conclusions}

In this paper we examine both quantitatively and qualitatively the large-scale structures in the Galactic disc. Two independent methods are applied to the 2MASS data. The first isolates red clump giants in a number of regions near the plane, the counts are then inverted to directly obtain the disc density. The second fits the parameters of a disc model in 820 independent nearplane regions. Results from both methods are consistent with each other implying that both methods are valid. For instance, magnitude and color of the red clump giants must be close to the correct values otherwise there would be significant difference between the two methods for $H$ and $h_{z}\left(R_{\odot}\right)$.

The distribution of sources within the disc is well described by an exponential distribution in both the galactocentric distance and height above the plane. There is a strong flare, i.e. an increase of scale-height towards the outer Galaxy. This flare starts well inside the solar circle, hence there is a decrease of the scale-height towards the inner Galaxy. This is consistent with the observation that the large majority of galaxies have flares (de Grijs \& Peletier 1997) and theoretically consistent with some scenarios of self-graviting stellar discs (Narayan \& Jog 2002). The flare removes the need to invoke a cut-off in the outer Galaxy, and certainly there is no evidence for an abrupt cut-off in the stellar disc, to at least $R<15 \mathrm{kpc}\left(=4.5 h_{R}\right)$. For the analysis it was asssumed that the red clump luminosity function could be described as a delta function and whilst it is shown that this could have some minor effect on the result, it could not mask a cut off.

In other spiral galaxies, the question of the cut-off is still not very clear. Pohlen et al. (2002) find that typical radial profiles are better described by a two-slope exponential profile, characterized by an inner and outer scale-length separated at a break radius, rather than a sharply-truncated exponential model. Florido et al. (2001), however, argue that there are some cases of abrupt truncation in the near infrared emission of the stellar profile. Kregel et al. (2002) finds that $60 \%$ of the spiral

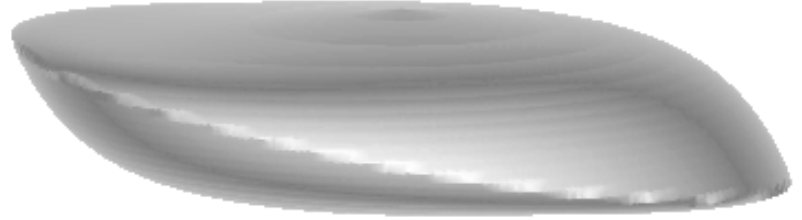

Fig. 23. Isodensity surface with $\rho=0.005 \mathrm{star} / \mathrm{pc}^{3}$ as described by the final disc model. Vertical scale multiplied by a factor 5 .

galaxies are truncated, although he also finds that the truncation is beyond $4.4 h_{R}$ for galaxies with $h_{R}<4 \mathrm{kpc}$; so probably, if there existed a cutoff in our Galaxy, it would not be detected in $R<4.5 h_{R}$.

Another noticeable feature is the warp in the old stellar population whose amplitude is coincident with the amplitude of the gas warp. Figures 22 and 23 show the form of the disc. Sánchez-Saavedra et al. (1990, 2002) or Reshetnikov \& Combes (1998) claim that most spiral galaxies are warped at optical wavelengths, but it is not easy to disentangle the stellar and gaseous warp.

Various parameters of the old stellar disc have been measured based on an analysis over galactocentric distances between $6 \mathrm{kpc}$ and $15 \mathrm{kpc}$. The study of the inner disc would be somewhat more complicate, especially in the plane, because the disc component is no longer isolated and it is mixed with other possible components: bulge, bar, ring, etc. As is shown in López-Corredoira et al. (2001), the disc might be a Freeman type II disc and inside about $3.5 \mathrm{kpc}$ the disc would be truncated; this deserves another detailed study which is beyond the scope of this paper. At present we can talk about the distribution at $R>5-6 \mathrm{kpc}$ and representative of the mean old disc at low latitudes (with mean $|z|$ around $300 \mathrm{pc}$ ). If there exists a thick disc, the results for higher latitudes would be substantially different. In the following are presented the results from the star counts, since they are potentially more accurate.

- The scale-height in the solar circle is $h_{z}\left(R_{\odot}\right)=285_{-12}^{+8} \mathrm{pc}$;

- the scale-length of the space density in the plane is $H=$ $1.97_{-0.12}^{+0.15} \mathrm{kpc}$;

- the scale-length of the surface density is $h_{R}=3.3_{-0.4}^{+0.5} \mathrm{kpc}$.

The errors do not include systematic uncertainties, which we know to be lower than a $10 \%$. For the flare and the warp:

- the variation of the scale-height due to the flare follows roughly a law $h_{z}(R) \approx h_{z}\left(R_{\odot}\right) \mathrm{e}^{\frac{R-R_{\odot}}{(12-0.6 R(\mathrm{kpc})) \mathrm{kpc}}}$ (for $R \lesssim 15 \mathrm{kpc}$ ); - the warp moves the mid plane of the disc to the height $z_{\mathrm{w}}=$ $1.2 \times 10^{-3} R(\mathrm{kpc})^{5.25} \sin \left(\phi+5^{\circ}\right)$ pc (for $R \lesssim 13 \mathrm{kpc}$ ), where $\phi$ is the galactocentric angle $\left(\phi_{\odot}=0\right)$.

The results presented here constitute an important step in constraining of the disc structure, especially for the stellar populations in outer disc, which were previously not very well known. Theoretical implications are not discussed here but it is expected that these results will constitute a valuable test for many theories about the formation and evolution of the galactic discs, as well as studies about the formation of warps, flares. 
Acknowledgements. This publication makes use of data products from 2MASS, which is a joint project of the Univ. of Massachusetts and the Infrared Processing and Analysis Center, funded by the NASA and the NSF. We gratefully acknowledge the anonymous referee whose detail report has helped improve the paper. Thanks are also given to $\mathrm{O}$. E. Gerhard, N. Bissantz and C. Alard for helpful comments.

\section{References}

Alard, C. 2000, preprint [astro-ph/0007013]

Bahcall, J. N. 1986, ARA\&A, 24, 577

Bahcall, J. N., \& Soneira, R. M. 1980, ApJS, 44, 73

Burton, W. B. 1988, in Galactic and Extragalactic Radio Astronomy, ed. K. I. Kellerman, \& G. L. Verschuur (Springer-Verlag, Berlin), 295

Buser, R., Rong, J., \& Karaali, S. 1998, A\&A, 331, 934

Buser, R., Rong, J., \& Karaali, S. 1999, A\&A, 348, 98

Cameron, L. M. 1985, A\&A, 147, 47

Cohen, M., Hammersley, P. L., \& Egan, M. P. 2000, AJ, 120, 3362

de Grijs, R., \& Peletier, R. F. 1997, A\&A, 320, L21

Diplas, A., \& Savage, B. D. 1991, ApJ, 377, 126

Djorgovski, S., \& Sosin, C. 1989, ApJ, 342, L13

Drimmel, R., \& Spergel, D. N. 2001, ApJ, 556, 181

Eaton, N., Adams, D. J, \& Gilels, A. B. 1984, MNRAS, 208, 241

Epchtein, N. 1997, in The Impact of Large Scale Near-IR Sky Surveys, ed. F. Garzón, N. Epchtein, A. Omont, B. Burton, \& P. Persi (Kluwer, Dordrecht), 15

Florido, E., Battaner, E., Guijarro, A., Garzón, F., \& Jiménez-Vicente, J. 2001, A\&A, 378, 82

Freudenreich, H. T. 1998, ApJ, 492, 495

Garzón, F., Hammersley, P. L., Mahoney, T., et al. 1993, MNRAS, 264,773

Gilmore, G., \& Reid, N. 1983, MNRAS, 202, 1025

Gyuk, G., Flynn, C., \& Evans, N. W. 1999, ApJ, 521, 190

Habing, H. J. 1988, A\&A, 200, 40

Hammersley, P. L., Garzón, F., Mahoney, T., \& Calbet, X. 1994, MNRAS, 269, 753

Hammersley, P. L., Garzón, F., Mahoney, T., \& Calbet, X. 1995, MNRAS, 273, 206

Hammersley, P. L., Cohen, M., Garzón, F., Mahoney, T., \& López-Corredoira, M. 1999, MNRAS, 308, 333
Hammersley, P. L., Garzón, F., Mahoney, T. J., López-Corredoira, M., \& Torres, M. A. P. 2000, MNRAS, 317, L45

Hoeg, E., Bässgen, G., Bastian, U., et al. 1997, A\&A, 323, L57

Kent, S. M., Dame, T. M., \& Fazio, G. 1991, ApJ, 378, 131

Kregel, M., van der Kruit, P. C., \& de Grijs, R. 2002, MNRAS, 334, 646

López-Corredoira, M., Hammersley, P. L., Garzón, F., Simonneau, E., \& Mahoney, T. J. 2000, MNRAS, 313, 392

López-Corredoira, M., Hammersley, P. L., Garzón, F., et al. 2001, A\&A, 373, 139

Majewski, S. R. 1993, ARA\&A, 31, 375

Narayan, C. A., \& Jog, C. J. 2002, A\&A, 390, L35

Ojha, D. K. 2001, MNRAS, 322, 426

Paul, E. R. 1993, in The Milky Way Galaxy and Statistical Cosmology, 1890-1924 (Cambridge University Press, Cambridge)

Pohlen, M., Dettmar, R. J., Luetticke, R., \& Aronica, G. 2002, in Disks of Galaxies: Kinematics, Dynamics and Perturbations, ed. E. Athanassoula, \& A. Bosma (ASP Conf. Ser., S. Francisco), in press, preprint [astro-ph/0201010]

Porcel, C., \& Battaner, E. 1995, MNRAS, 274, 1153

Price, S. D. 1988, PASP, 100, 171

Reid, M. J. 1993, ARA\&A, 31, 345

Reshetnikov, V., \& Combes, F. 1998, A\&A, 337, 9

Rieke, G. H., \& Lebofsky, M. J. 1985, ApJ, 288, 618

Ruphy, S., Robin, A. C., Epchtein, N., et al. 1996, A\&A, 313, L21

Sánchez-Saavedra, M. L., Battaner, E., \& Florido, E. 1990, MNRAS, 246, 458

Sánchez-Saavedra, M. L., Battaner, E., Guijarro, A., LópezCorredoira, M., \& Castro-Rodríguez, N. 2002, A\&A, submitted

Sarajedini, A., Lee, Y. W., \& Lee, D. H. 1995, ApJ, 450, 712

Skrutskie, M. F., Schneider, S. E., Stiening, R., et al. 1997, in The Impact of Large Scale Near-IR Sky Surveys, ed. F. Garzón, N. Epchtein, A. Omont, B. Burton, \& P. Persi (Kluwer, Dordrecht), 25

van der Kruit, P. C. 1988, A\&A, 192, 117

Wainscoat, R. J., Cohen, M., Volk, K., Walker, H. J., \& Schwartz, D. E. 1992, ApJS, 83, 111

Wouterloot, J. G. A., Brand, J., Burton, W. B., \& Kwee, K. K. 1990, A\&A, 230, 21 